\title{
Espacialidad local e inka en el Valle Calchaquí Norte (Salta, Argentina): Reevaluando el alcance de la INTERVENCIÓN IMPERIAL EN LA PAYA
}

\author{
Alejandro Andrés Ferrari ${ }^{1}$
}

\begin{abstract}
Resumen
En este artículo presentaré ciertas características del sitio arqueológico La Paya (SSalCac 1), ubicado en el Noroeste Argentino; más precisamente en el Valle Calchaquí Norte. Se trata de un asentamiento que presenta ocupaciones del Período Intermedio Tardío e intervenciones arquitectónicas correspondientes al Período Inka. Éstas consisten en una serie de remodelaciones entre las que se incluyó la construcción de una estructura de neta filiación Inka conocida como Casa Morada, cuyas características arquitectónicas y localización han sido conceptualizadas como intrusivas respecto de la arquitectura local. A continuación, repasaré aquellas investigaciones y presentaré nuevos datos para enriquecer el entendimiento de estas intervenciones. Argumentaré que la intervención Inka involucró más estructuras que las que fueron previamente señaladas, trastocando así el modo de habitar local, demarcando la diferencia entre colonizadores y colonizados, y cristalizando nuevos segmentos dentro de la población local.
\end{abstract}

Palabras clave: Período Intermedio Tardío - Período Inka - Noroeste Argentino - Arquitectura - Dominio Inka

In this paper, I will present certain characteristics of an archaeological site known as La Paya (SSalCac 1), located in northwest Argentina; more precisely in the North Calchaqui Valley. It features occupancies during the Late Intermediate Period and architectonical interventions belonging to the Inka Period. The latter consist of a series of remodelations, including the construction of an Inka structure known as Casa Morada, whose architectural properties have been conceptualized as intrusive regarding local layouts and construction techniques.

Below, I summarize previous investigations and introduce new data to enrich our understanding of these interventions. I will argue that imperial interventions involved more structures than those previously reported, disrupting the local way of dwelling, demarcating the difference between colonizers and the colonized, and crystallizing new segments within the local population.

Key words: Late Intermediate Period - Inka Period - Northwest Argentina - Architecture - Inka rule

Recibido: Septiembre 2014. Aceptado: Abril 2016

\section{* Introducción}

La ocupación del Valle Calchaquí Norte (Salta, Argentina) (Figura 1) se vio afectada, hacia $1450 \mathrm{DC}$, por el arribo de los Inkas y el despliegue de espacialidades y materialidades que trastocaron y permearon variablemente las esferas de la vida social local.

En este artículo presentaré ciertos datos y apreciaciones respecto de Casa Morada y el complejo de estructuras que la alberga. La Paya (SSalCac 1) es un poblado local cuya ocupación corresponde predominantemente al Periodo Intermedio Tardío, con fechados que abarcan desde

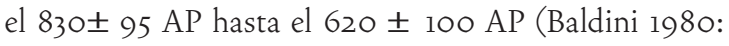
60). Luego del arribo de los representantes imperiales, se emplazó una estructura de neta filiación Inka conocida como Casa Morada en un sector particular del asentamiento. Ésta, junto con el complejo arquitectónico en que se emplaza, ilustra el modo en que los Inkas crearon nuevas condiciones para la espacialidad local y direccionaron la experiencia de lo foráneo.

Dado que la arquitectura de Casa Morada y ciertas estructuras que la rodean ha sido estudiada en diferentes oportunidades por diferentes investigadores e investigadoras (Ambrosetti 1907; Boman 1908; Alfaro de Lanzone 1985 y, fundamentalmente, González y Díaz 1992), no me ocuparé aquí en detalle de las técnicas constructivas empleadas (ampliamente descriptas en estos trabajos) más allá de aquellos aspectos que hacen a la circulación interna del sector en que se halla.

1 CONICET - Instituto de Estudios Americanistas Julián Cáceres Freyre (Universidad Austral). Dirección postal: Cerrito 1250 - Ciudad Autónoma de Buenos Aires, Argentina. Correo electrónico: alejandroferra@gmail.com 


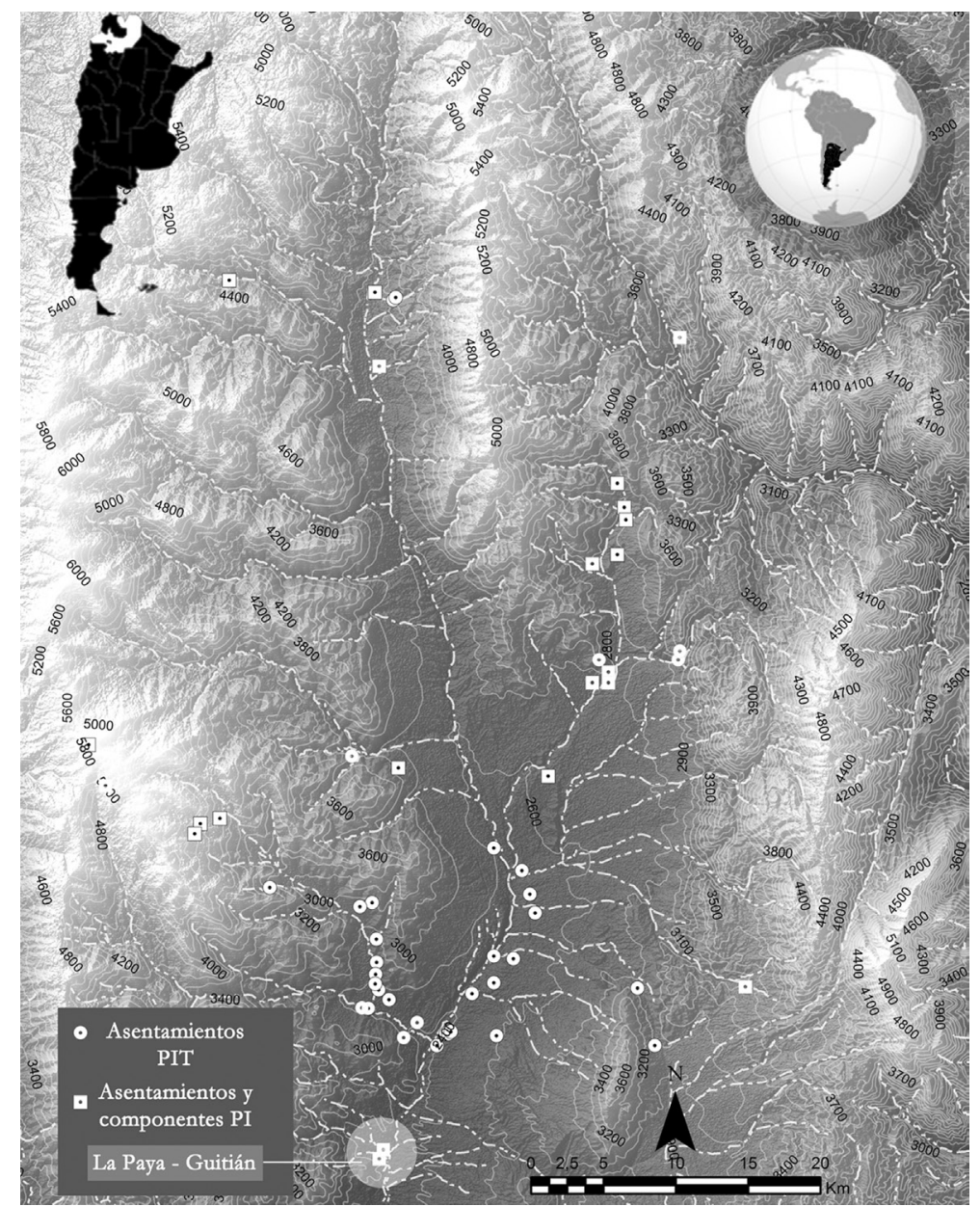

Figura 1: Valle Calchaquí Norte y ubicación de La Paya y Guitián.

El eje principal que va a guiar este artículo contempla, en primer lugar, los elementos que caracterizan la circulación y accesibilidad dentro de La Paya antes de la intervención inkaica. Enfatizaré la importancia de la distribución de las principales vías de circulación y el modo en que crean sectores discretos, los conectan, y dirigen la accesibilidad hacia su interior. En segundo lugar, llamaré la atención sobre la circulación que habilitan las técnicas constructivas empleadas tanto en las estructuras locales como en el sector en que se encuentra el complejo de estructuras que alberga a Casa Morada. Argumentaré, finalmente, que la intervención en La Paya no se limitó al emplazamiento de una estructura de neta filiación Inka en un poblado local y la reorganización parcial de ciertos recintos, sino que implicó rediseñar la totalidad del complejo de estructuras que la alberga comenzando por su accesibilidad desde las principales vías de circulación del sitio. Es decir, que implicó más modificaciones de las que fueran previamente señaladas.

\section{MARCO TEÓRICO GENERAL}

La arquitectura y el modo en que reorganiza el terreno, canaliza el movimiento de los cuerpos y las vistas, y crea determinadas condiciones de posibilidad para la interacción interpersonal, ha sido objeto de estudio de varias disciplinas y temáticas. Esta conceptualización general, que ancla a la arquitectura a su papel de productora, reproductora y por ello directriz de la vida social, resulta de particular importancia cuando se evalúa la creación de condiciones de participación en espacialidades cuyo objeto es crear órdenes específicos que guían la construcción del mundo y de las subjetividades (Thomas 1993; Hayden 1997). 
Esto implica un desplazamiento general que invita a repensar el modelo de espacio moderno (universal, constante y abstracto) para desplazarlo hacia un espacio múltiple, socialmente producido, historizado, y producto a la vez que transformador del espacio físico y psicológico (Soja 1989). Estos desplazamientos marcan una dirección general: nos alejan del aspecto estrictamente mental y racional del proceso de conocimiento y nos llevan a la intersubjetividad, la experiencia, la percepción y al cuerpo como fuente de conocimiento y constitución de la subjetividad. Es decir, hacia un sujeto socialmente informado y un espacio socialmente producido.

\section{Hacia un sujeto socialmente informado}

Partiendo de que toda percepción es el producto de un proceso interpretativo en el que la percepción presente está conectada con percepciones pasadas previamente experienciadas, nuestra percepción en el mundo es abierta e inexhaustible en tanto que el sujeto que percibe no es impermeable a nuevas situaciones naturales e históricas. Es decir, el sujeto que percibe no lo hace de acuerdo a un esquema de pensamiento transparente y accesible para y por él mismo, sino que se encuentra influenciado por su biografía experiencial (Merleau Ponty 1962; Dilley 1999; Van Dyke y Alcock 2003).

Desplazando el eje de la percepción hacia la práctica, los agentes no conocen el mundo mediante una relación de exterioridad producto de una conciencia conocedora (es decir, con la distancia objetivadora de un modelo de sujeto racionalista crítico) sino que lo comprenden toda vez que se encuentran inmersos en él, habitándolo pre objetivamente como un hábito o un hábitat familiar (Csordas 1990; Bourdieu 2007). En este esquema, el cuerpo opera como "...una memoria a la que se confian, de un manera abreviada y práctica, es decir mnemotécnica, [...] los principios fundamentales del contenido arbitrario de la cultura." (Bourdieu 1977: 94, traducción del autor). De aquí que el cuerpo deba ser entendido como una potencia inmersa en la historia, con una potencia creativa que habilita una libertad limitada; la producción de estructuras estructurantes durante la reproducción de lo estructurado. En palabras de Bourdieu: "El cuerpo cree en aquello a lo que juega: llora si imita [en una relación global de identificación] la tristeza. No representa aquello a lo que juega, no memoriza el pasado, actúa el pasado, anulado así en cuanto tal, lo revive. Lo que aprende uno con el cuerpo no es algo que uno tiene, como un saber [...] sino algo que uno es" (2007:118).

El movimiento en sí es también una práctica del pensar que se nutre de nuestra inmersión corporal en el mundo (Thomas 2001) e implica la adquisición de un "saber ambulatorio" (Ingold 2010: 122) que a su vez, en la práctica, comunica a través de saberes performativos no discursivos (Thomas 2002; Strathern 2009). Este proceso se inserta en un proceso mayor, comunicativo, que implica un aprendizaje por participación, en acción, y de otro orden respecto del aprendizaje expositivo y verbalizado (Lave y Wegner 1991; Turner 1994; Knappett 2005; Budden y Sofaer 2009).

Si acordamos que el comportamiento corporal conlleva siempre un elemento histórico, que por ello nuestro cuerpo opera como depositario y comunicador de convenciones sociales trascendiendo su fisicalidad (Ingold 2000), que el conocimiento por cuerpos no implica únicamente un aprendizaje cognitivo - reflexivo consciente sino un ejercicio imitativo incentivado por la cotidianeidad (Bourdieu 2007), y que la vida social está hecha de acción corporal competente en contextos particulares (Gosden 2002), entonces las transformaciones del espacio físico y el modo en que organiza y coordina las actividades cotidianas opera como un instrumento capital a la hora de crear identidades, comunicar posiciones en la estructura social, y dirigir su reproducción.

\section{Hacia espacios socialmente producidos}

Como producto social, el espacio está sujeto a la estructuración espacio temporal de la vida social de modo que opera como "...medio y resultado, como presuposición y corporeización, de la acción y relación social" (Soja 1989:129, traducción del autor). Estas dualidades del espacio socialmente producido lo involucran necesariamente en prácticas sociales imbricadas en luchas por la producción y reproducción social, ya sea destinadas a la manutención y refuerzo de la espacialidad existente o a su reestructuración. Concebir el espacio como producto socializado implica fundamentalmente que el mismo no puede ser experienciado de modo neutral (esto es, significado de acuerdo a las propiedades del espacio físico o natural sin intervención de la influencia de la historicidad de los sujetos), sino constantemente leído en función de los 
espacios que se le han revelado previamente al sujeto (Gosden y Head 1994).

Esto implica que, en tanto que narrativas históricamente construidas, pueden estar sujetas a modificaciones producto de la reorganización del movimiento corporal arquitectura mediante, o bien en función de la coexistencia con otras narrativas espaciales. Aquí es que cobran especial relevancia aquellos espacios que se encuentran estructurados o presentados de modo tal que crean diferentes arenas de la práctica corporal, o bien que son alterados de modo tal que presentan rupturas con modos precedentes de habitar y relacionarse con otros miembros de la comunidad (Bell 1992; Turner 1994).

La arquitectura y su capacidad para diagramar la movilidad y por ello ordenar los cuerpos, las vistas, las cosas (sensu Ingold 2010b) con las que entramos en relación, opera como una herramienta de particular relevancia cuando se examinan las condiciones de posibilidad para crear accesos parciales a distintos recursos. Transformar el espacio, dependiendo del contexto, puede estar orientado tanto controlar el acceso a recursos básicos de subsistencia como a asegurar el acceso parcial a diferentes tipos de información (Nielsen 1995). Segmentar grupos o sujetos, condicionar su interacción, y configurar la exposición a las performatividades que los informan, se revela como uno de los modos cruciales en los que a los ambientes construidos despliegan su potencial como productores y reproductores de procesos identitarios.

\section{La incorporación del Valle Calchaquí Norte AL IMPERIO INKA}

Pese a la cantidad y variedad de investigaciones respecto del Imperio Inka, un enunciado general en el que confluyen varios investigadores es que los asentamientos inkaicos operaron como representaciones de un sistema institucional político y económico que se adaptó a los medios sociales y económicos de las áreas conquistadas (Earle y D'Altroy 1989; Williams y D'Altroy 1998; D'Altroy 2002, ver Burguer et al. 2007, entre muchos otros). Los grados de uniformidad arquitectónica y artefactos presentes, pese a la variabilidad ambiental y cultural de las zonas anexadas, dan cuenta de la participación común en tareas y enclaves cuya construcción y desarrollo estuvo dirigida o al menos incentivada por el impe- rio (Gasparini y Margolies 1980; Raffino 1981; Hyslop 1990; D’Altroy 1992; Moore 1996; Covey 2015).

Se ha demostrado que la política Inka para esta porción de los Andes meridionales articuló una serie de actividades fundamentales que se reflejan en la infraestructura básica de ocupación imperial: la instalación de fortalezas en las fronteras para proteger nuevos territorios y disuadir rebeliones internas, la instalación de centros estatales (ya sea enclaves de producción en valles fértiles o enclaves administrativos en zonas estratégicas de comunicación y control) y la construcción sitios para propósitos especiales, postas de enlace, adoratorios de altura, y una extensa red de caminos principales y secundarios que articulaban todos ellos (Hyslop 1984; Williams y D'Altroy 1998; Williams 2000). El objetivo detrás de esta distribución de enclaves, junto con la intensificación de la producción agropastoril, minera, artesanal, y el reasentamiento de personas con el objetivo de administrar los territorios, fue el aprovisionamiento de mano de obra y recursos para el imperio (Raffino 1978, 1981; Williams 1991, 1994, 2000, 2004; D'Altroy et al. 2000, entre otros).

En el Valle Calchaquí Norte, la estrategia de comunicación de la cosmovisión imperial estuvo orientada primordialmente hacia la escenificación y despliegue de espacialidades y materialidades junto con la manipulación, control e incentivo de la participación local en prácticas y espacios ritualizados que, en conjunto: demarcaban el contraste entre colonizadores y colonizados, creaban un nuevo nivel organizativo por arriba de las unidades políticas locales, y reorganizaban el paisaje local promoviendo nuevas representaciones y transformando los sentidos de lugar (Acuto 1999; 2010). Testigo de ello es la reorganización de ciertos asentamientos (como ser el complejo de Cortaderas, ver en Acuto y Gifford 2007), la construcción de adoratorios de altura y circuitos de peregrinaje hacia las principales cumbres nevadas de la región (Ceruti 2003; Vitry 2008; Jacob y Leibowicz 2011), el emplazamiento de pequeños centros en la inmediatez de antiguos poblados (como ser Guitián, a tan sólo 345 m de La Paya, Figura 2), y la intrusión de estructuras y/o complejos arquitectónicos dentro o en la inmediatez de poblados locales (ver en González y Díaz 1992; Gifford 2003).

La reorganización de asentamientos locales no es exclusiva de esta región, sino que se ha documentado para 


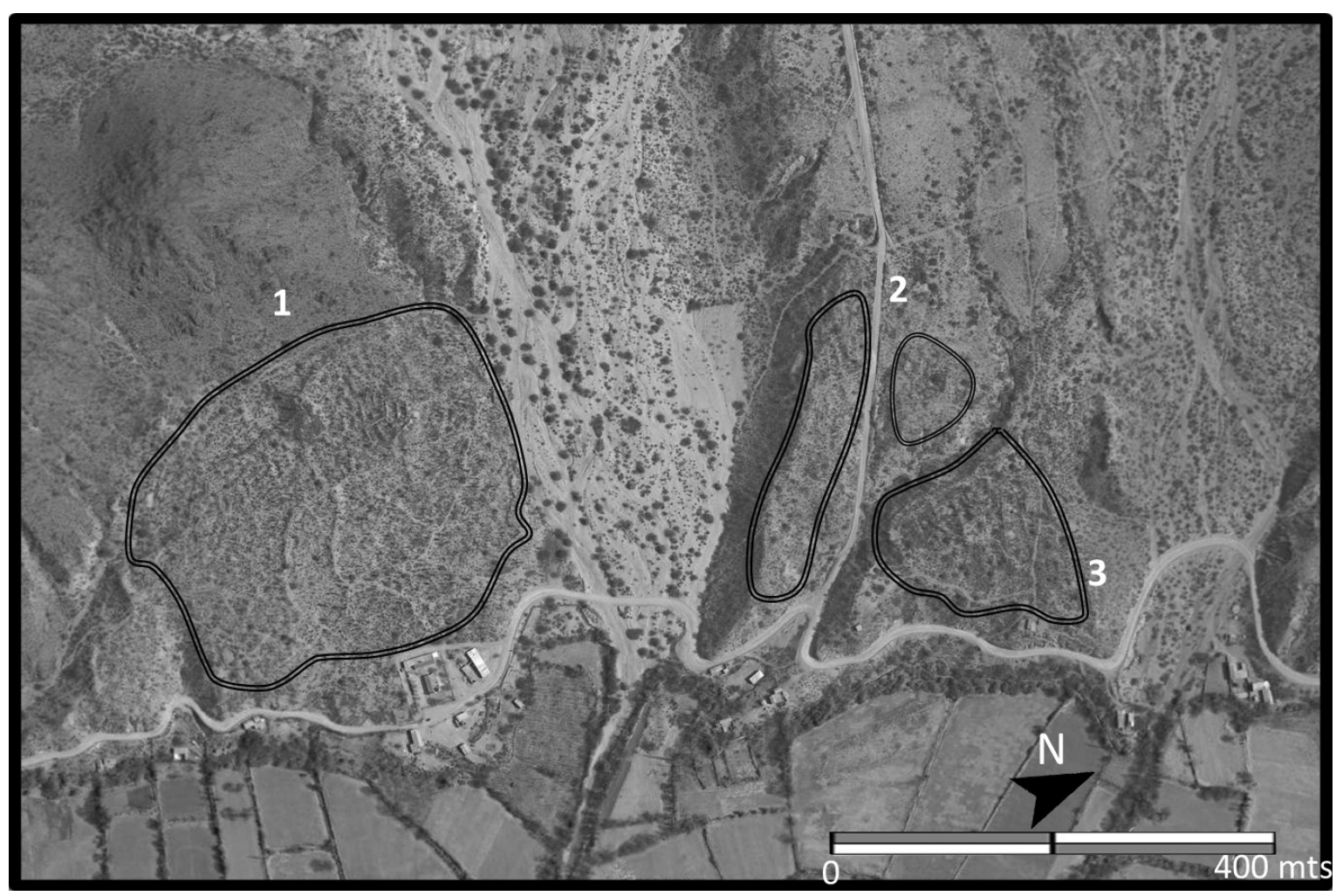

Figura 2: La Paya (1), Guitián (3) y asentamiento no registrado aún (2). Imagen tomada de Google Earth.

otras zonas del imperio (ver, por ejemplo, Morris y Santillana 2007; Kosiba 2010) y otras regiones del Kollasuyu, como ser la Quebrada de Humahuaca. Allí, en el sitio Los Amarillos (ver en Nielsen 1995; Nielsen y Walker 1999; Nielsen 2006) el componente Inka no sólo se emplazó en donde había un complejo arquitectónico ceremonial previo en la sección central más elevada del asentamiento, sino que se modificó su diseño de planta para ocultar las actividades realizadas. Esta reorganización, pese a que implicó el aprovechamiento parcial de los antiguos muros, ignoró mayormente el antiguo trazado, y convirtió un espacio destinado a ceremonias de carácter público en un espacio doméstico y privado para un grupo estrechamente vinculado al imperio (Nielsen y Walker 1999: 166). Otro ejemplo notable es el de La Huerta (ver en Leibowicz 2007, 2011), también en la Quebrada de Humahuaca, en donde se emplazó un complejo arquitectónico que, si bien no se han detectado ocupaciones previas, presenta un control exhaustivo de su accesibilidad física y claros indicios de que un muro impedía ver lo que allí sucedía desde los sectores vecinos.
A su vez, y al igual que en otras regiones del imperio, los Inkas establecieron alianzas estratégicas con ciertas comunidades y grupos locales. Varios estudios sobre la colonización Inka a lo largo de los Andes muestran el modo en que ciertos grupos aprovecharon su relación con el Tawantinsuyu, emulando la cultura material inkaica para mejorar su posición y transformar y legitimar su estatus (Grosboll 1993; Mackey 2003; Alconini 2010).

\section{La Paya: Antecedentes}

La Paya (SSalCac1), es un sitio conglomerado de aproximadamente seis hectáreas rodeado por un muro perimetral de $1239 \mathrm{~m}$ de recorrido (Ambrosetti 1907: 36). Comparte algunas características generales con otros sitios del Período Intermedio Tardío en la región: está ubicado en un piedemonte (sobre una terraza adyacente al río homónimo) y presenta mayoritariamente conjuntos de estructuras semisubterráneas entre las cuales la circulación era posible ya sea mediante accesos directos entre estructuras, mediante rampas de acceso a las estructuras, 
o bien sobre la superficie caminable entre los muros de cada estructura (a estos últimos los llamaré "espacios interedilicios" despejados de ahora en más²). Los tipos de estructuras, técnicas constructivas, y articulación entre estructuras son similares a otros sitios asociados al Período Intermedio Tardío en la región.

Las investigaciones aún son pocas para un sitio de esta envergadura. Fundamentalmente han estado dirigidas a: la recuperación de materiales de contextos domésticos (Díaz 1981), mortuorios (Ambrosetti 1907), el estudio de artefactos cerámicos a fin de determinar estilos locales (Ambrosetti 1902; Boman 1908; Bennett et al. 1948; Calderari 1991) y variaciones cronológicas (Serrano 1963; Baldini y Balbarrey 2004), su correlación estilística y cronológica con cerámicas provenientes de otros sitios (Tarragó y De Lorenzi 1976; Baldini 1980; Alfaro de Lanzone 1985; Calderari 1991; Baldini y Sprovieri 2009), diferencias e hibridaciones entre estilos locales e Inka (Bennett et al. 1948; Calderari 1991; Calderari y Williams 1991), y estudios relativos a la composición y manufactura de objetos metálicos (González 2003). Adicionalmente, han resultado de vital importancia para el estudio de la interacción interregional entre el Noroeste Argentino y el Norte de Chile (Sprovieri 2008 - 2009) y entre el Valle Calchaquí y las yungas (Sprovieri y Rivera 2014). Es decir que el grueso de las investigaciones ha estado dirigido al análisis de los materiales extraídos en excavaciones con el objeto de asignar correlaciones estilísticas, cronologías absolutas y relativas, determinar el alcance de las interacciones regionales $y$, en menor medida (ver en Díaz 1981; Alfaro de Lanzone 1985; González y Díaz 1992), a las estructuras presentes en general.

El estudio de los materiales extraídos en las excavaciones de Ambrosetti ha sido utilizado también para realizar estudios referidos a prácticas mortuorias en general (Amuedo 2010; Acuto et al. 2011), a la corroboración y discusión de los derivados materiales de los modelos que generaron la interpretación clásica del desarrollo cultural del Noroeste Argentino (ver en Acuto 2007; Acuto et al. 2008) y a continuidades y discontinuidades respecto de la manufactura de artefactos cerámicos para el intervalo temporal que abarca desde el Período Intermedio Tardío hasta el Período Hispano Indígena (Gamarra 2008). Estos estudios permitieron proponer alternativas respecto de la interpretación tradicional de la orga- nización social de estas comunidades.

El primer trabajo de investigación de importancia publicado respecto de La Paya fue el producto de dos campañas, financiadas por la Facultad de Filosofía y Letras de la Universidad de Buenos Aires, llevadas adelante en 1906 y 1907 por Juan Bautista Ambrosetti. Realizó una descripción somera de algunas características fundamentales de la arquitectura y de su distribución. Describió estructuras "...formando grandes canchones o cuadrados de cuatro $y$ cinco metros de ancho porocho o diez de largo, colocados por series de seis o siete uno al lado del otroparalelamente dispuestos" (1907:38); para las que "...se han aprovechado los desniveles del suelo y además se han completado con trabajos de cava en muchos puntos para proporcionarse áreas subterráneas o sótanos..." (1907:40). En estas áreas excavadas, sus superficies verticales "...se han sostenido por una pirca (I) de piedra para impedir el desmoronamiento" (1907:41). Posteriormente, el relevamiento llevado adelante por Díaz (1981), además de haber realizado numerosas excavaciones en uno de los sectores del sitio (Sector 2 en Figura 3), ha señalado tres aspectos fundamentales: la predominancia de estructuras subrectangulares, la ausencia de vértices en ángulo recto, y la ligera curvatura de los muros que componen las estructuras. Por su parte, el relevamiento que llevó a cabo Alfaro de Lanzone (1985) señala la existencia de siete tramos y seis senderos principales, e interpreta que los caminos interiores "limitan verdaderos barrios o sectores" (1985:566). A continuación, se expone las características principales de la circulación al interior del sitio ${ }^{3}$.

2 Denominación por la que he optado y que, en líneas generales, se correspondería con lo que González y Díaz (1992: 29) han denominado "pared-sendero". Sin embargo, como se verá más adelante, el hecho de estar algunos de ellos bloqueados amerita un término más neutral a propósitos expositivos.

3 Todos los datos respecto de los desniveles aquí presentados derivan directamente de la lectura de las cotas desde el nivel óptico. Respecto del ancho de las vías de circulación, de los accesos, y de los espacios interedilicios, todas las medidas fueron tomadas in situ. En el caso de las vías de circulación, se tomaron medidas a intervalos regulares de seis metros allí en donde se detectaron superficies niveladas o bien muros de contención laterales a la vía de circulación pero que no forman parte del perímetro interno de las estructuras. Las diferencias de nivel presentadas aquí respecto de las vías de circulación y el interior de los sectores, son el resultado de las diferencias mínimas y máximas desde la superficie de las vías de circulación hasta la superficie interna y 


\section{Circulación Intrasitio}

La Paya está surcada por dos vías o senderos principales de circulación que cortan el sitio en sentido Norte Sur y lo seccionan en tres niveles topográficos distintos (Figura 3). De estas vías de circulación se desprenden una serie de senderos subsidiarios hacia el Este y el Oeste que terminan por delimitar la superficie de los sectores y conectar, aunque no directamente, todos ellos. Por lo pronto, basta destacar que el ancho de una de ellas (sendero principal $\mathrm{n}^{\circ} 2$ en figura 3) oscila entre 2,3 y 4,4 m $(\bar{X}=3,87 \mathrm{~m}$ ) a lo largo de $244 \mathrm{~m}$ de recorrido (llegando a $6 \mathrm{~m}$ en aquellos tramos en que confluye con senderos secundarios) y, la que reviste especial importancia para este trabajo (sendero $\mathrm{n}^{\circ} \mathrm{l}$ en figura 3 ), mantiene una media de 2,71 $\mathrm{m}$ en sus $217 \mathrm{~m}$ de recorrido, con un ancho máximo de $6 \mathrm{~m}$, y o, $6 \mathrm{~m}$ en su punto de máxima angostura.

Las vías de circulación principales presentan bifurcaciones hacia el Este y el Oeste. Estos senderos auxiliares (o secundarios) circunvalan conjuntos discretos de estructuras a los que se ha denominado sectores. Se identificó un total de 17 sectores, 16 de ellos compuestos únicamente por estructuras locales (Figura 3). Adicionalmente, estos senderos auxiliares dirigen la accesibilidad al interior de los sectores, ya sea a través de la circulación interedilicia o hacia senderos internos. El hecho que los senderos principales y secundarios circunvalen los sectores, junto con la predominancia de la circulación interedilicia, implica que existían múltiples accesos a ellos. Es decir, al menos toda vez que exista un claro sendero que sale de la vía de circulación y se dirija al interior del sector ( $\mathrm{Fi}$ gura 4) en donde confluye con otros senderos internos y/o toda vez que confluyan los senderos principales o secundarios con dos estructuras contiguas, dispuestas paralelamente, y con sus espacios interedilicios despejados.

Adicionalmente, estos sectores se encuentran circunvalados al menos en tres de sus lados por senderos cuya elevación actual respecto de la superficie interna de las estructuras dentro de ellos oscila actualmente entre 0,4

actual de las estructuras. De todos modos, dada la deficiente conservación del asentamiento y los procesos postdepositacionales que lo afectan, se aconseja consultar los perfiles topográficos realizados por Alfaro de Lanzone (1985) hace poco más de 30 años.

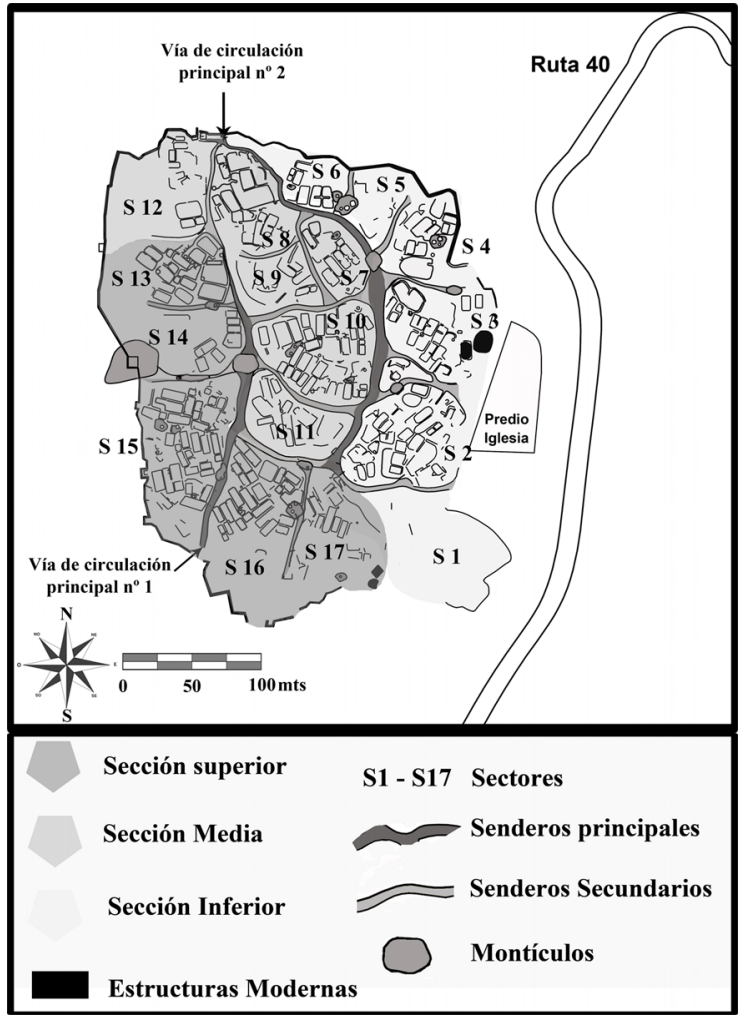

Figura 3: Plano general de la superficie intramuros de La Paya (tomado y modificado de Acutoet al. 2012)

y 5,4 m. Es decir que, durante su recorrido, y dada la elevación relativa respecto de la superficie interna actual de las estructuras, habilitaba a los transeúntes a visualizar lo que ocurría en el interior de aquellas estructuras que no hubiesen estado techadas. Se ha señalado (ver Gifford 2003: 242 - 333) que la mayoría de las estructuras que componen los conglomerados tardíos podrían no haber estado completamente techadas o no haber estado techadas en absoluto. A lo sumo, con horcones lo largo del perímetro interno de las estructuras dejando un espacio libre y no techado en el medio (Ambrosetti 1907). Sin embargo, pese a que esto debe ser adecuadamente contrastado, me permito inferir que la techumbre estaba tan estandarizada como los tipos edilicios presentes. Los relevamientos realizados en los últimos años me permitieron definir, en base a la geometría de las plantas de las estructuras locales, un lenguaje observacional que consta 
de 18 tipos edilicios ${ }^{4}$. De estos 18 tipos edilicios presentes en el asentamiento, tan sólo tres de ellos (subcuadrangulares, subrectangulares, y circulares) representan el $74,8 \%(n=187)$ de las estructuras cuyo perímetro es claramente discernible. Adicionalmente, la distribución de la totalidad de las estructuras entre los sectores estrictamente locales mejor conservados no exhibe diferencias estadísticamente significativas $\left(\mathrm{X}_{2}=181,87 ; \mathrm{gl}=153 ; \mathrm{p}=\right.$ $0,06)$ que permitan aislar alguno de ellos (Ferrari 2012).

El primer aspecto que derivo de esta distribución de vías de circulación es que, en conjunto, garantiza iguales condiciones de accesibilidad física a cualquiera de los sectores estrictamente locales del sitio. Los sectores deben ser siempre circunvalados al menos por uno de sus senderos limítrofes para acceder a su interior y, aún el sector 15 (delimitado por el muro perimetral y sólo 2 vías de circulación) no presenta dificultad alguna a la hora de llegar a él.

Por el estado actual de conservación de la muralla perimetral, resulta muy arriesgado diagramar un modelo de circulación en función de la cantidad potencial de entradas al sitio e inferir qué sectores debían ser necesariamente circunvalados (aunque sea parcialmente) para llegar a otro. Sin embargo, la clara diferencia en el ancho de las vías de circulación principales, secundarias, e intrasectoriales $(0,6$ a 1,2 $\mathrm{m}$ ) me indican que eran funcionales a la cantidad de personas que por allí circulaban. Esto señala uno de los aspectos principales a destacar: además de constituir el modo fundamental de acceder a los sectores, los espacios interedilicios son los elementos fundamentales que guían la circulación en su interior.

4 Subrectangular, subrectangular con división transversal, subrectangular con división longitudinal, subrectangular con división interna, subrectangular con apéndice, subrectangular abierta, circular en espacio interedilicio, circular en montículo, circular interna, subrectangular interna, subcircular, semicircular interna, elipsoidal, subcuadrangular, adjunta al muro perimetral con proyección interna, adjunta al lienzo interno de la muralla perimetral, triangular, y perímetro variable.

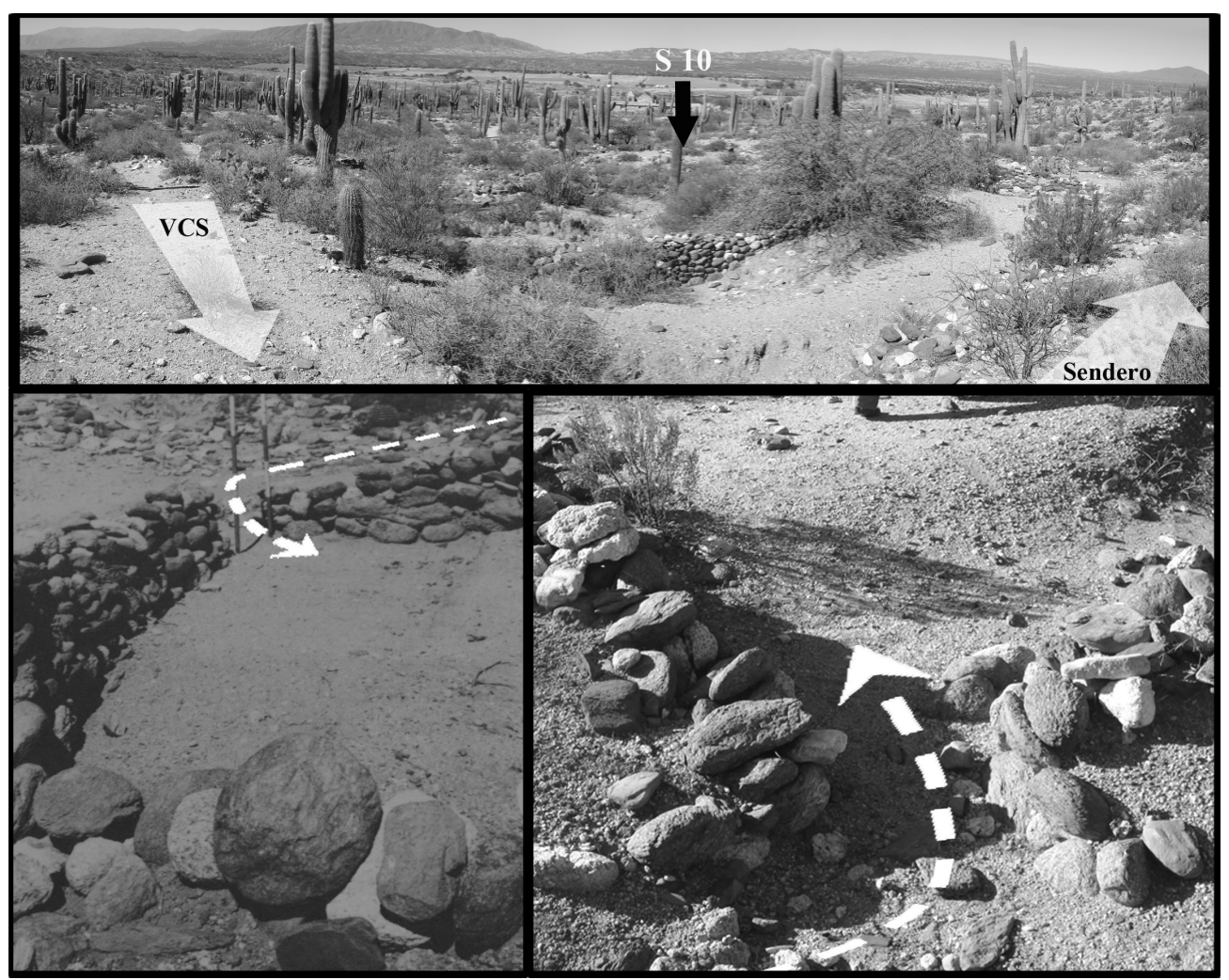

Figura 4: Ejemplo de accesibilidad a un sector por espacio interedilicio desde Vía de Circulación Secundaria (VCS) y posterior ingreso a estructura por sendero interno.La fotografía inferior izquierda fue tomada de Alfaro de Lanzone(1985:583). 
Esto implica un diseño de articulación local que preveía la circulación de personas por todo el sitio.

En definitiva, la distribución de los senderos principales y secundarios junto con las técnicas constructivas empleadas en las estructuras, estaban diagramadas de modo tal que, en primer lugar, aseguraban la accesibilidad de los 16 sectores estrictamente locales. En segundo lugar, que arribar a un sector del sitio implicara necesariamente bordear otros sectores locales. Finalmente, que transitar estos senderos, que a su vez creaban múltiples condiciones de acceso al interior de los sectores, promoviera la visualización de su interior y posiblemente del interior de aquellas estructuras no techadas o parcialmente techadas. Quien recorriese La Paya, independientemente del sector local al que pretendiese acceder, participaba regularmente de los mismos eventos performativos y similares condiciones de posibilidad para la inteligibilidad de aquellos. Recorrer implicaba ver y conocer lo que sucedía en la comunidad. El modelo histórico particular de sujeto local se nutría de la observación de y participación en una cotidianeidad que no buscaba distinguir ni restringir, sino redundar, homogeneizar y favorecer la comunicación e interacción interpersonal en función de la repetición de espacios y actividades. Ahora bien, iqué ocurre con la accesibilidad física y visual de aquel sector en el que se emplazó Casa Morada?

\section{Arribando al sector 13: Casa Morada}

Casa Morada (Figura 5) fue descripta por J. B. Ambrosetti (ver Ambrosetti 1907) como una estructura rectan- gular de 13,9 m x 4,3 $\mathrm{m}$ con muros de 3,4 $\mathrm{m}$ de altura y $0,69 \mathrm{~m}$ de espesor construidos con bloques tabulares de arenisca roja, y con un único acceso de 1,21 m la sección media de su lado Norte. Señaló también la presencia de cuatro nichos de 0,4 $\mathrm{m} 2$ espaciados a 1,1 $\mathrm{m}$ en su lado Sur. Interpretó que allí debió haber residido el "cacique local" o alguien de suma importancia 5 . Posteriormente, el trabajo de González y Díaz (1992), además de ofrecernos el primer croquis detallado de las estructuras que rodean Casa Morada (en Figura 5), nos ofrece descripciones detalladas de su estado de conservación y de las estructuras que la rodean. Limitaré ciertas referencias a aspectos que revisten especial interés para este artículo. Uno de estos aspectos es el acceso de Casa Morada, el cual "... oscila entre 1,18 y 1,23 m. No hallamos umbral de ninguna clase y en la poca altura conservada no pudo observarse variante del ancho, lo que nos hace suponer que su perímetro fue rectangulary no trapezoidal. Nada dicen respecto de este perímetro los artículos

5 Interpretación compartida por González y Díaz quienes agregan que "...la C.M. fue vivienda y centro administrativo al mismo tiempo." (1992:30) y que "...caben pocas dudas de que la Casa Morada fue la vivienda el curaca local."(1992:43). Adicionalmente, se habrían hallado adornos de oro, bronce aleado con estaño, artefactos de madera, hueso, y cerámica (ver Ambrosetti 1907: 48 -51). Debo aclarar, sin embargo, que el origen de estos materiales resulta confuso y que no existen registros fiables para su asignación (para ver una detallada descripción de este aspecto y discusiones respecto de su origen, ver Boman 1908; González y Díaz 1992, González 2003). La altura potencial de Casa Morada también ha sido un aspecto discutido. De todos modos González y Díaz (1992: 43) concluyen que “...la descripción más exacta fue la de J.B. Ambrosetti...".

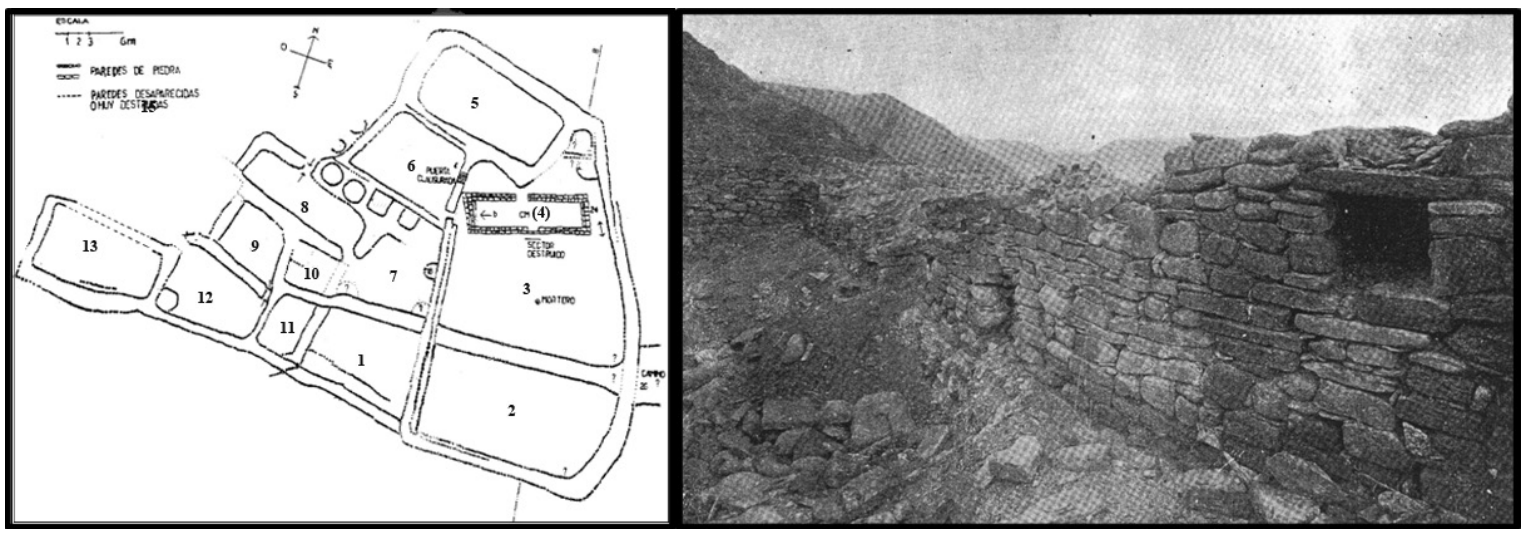

Figura 5: Izquierda: Primer croquis detallado de Casa Morada (ligeramente modificado de González y Díaz 1992). Derecha: estado de conservación hacia principios del Siglo XX (tomada de Ambrosetti 1907:46). 


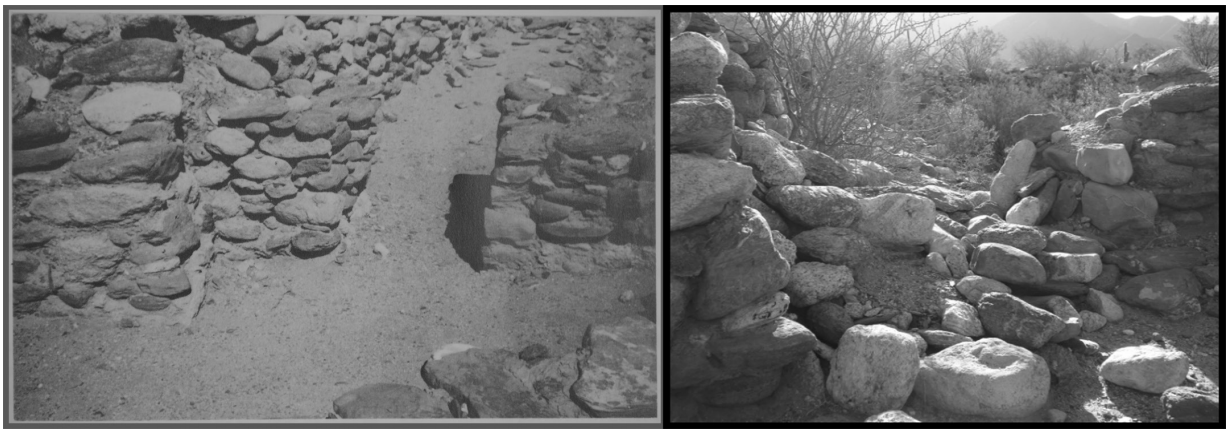

Figura 6: Izquierda: Fotografía del acceso que comunica las estructuras 3 y 6 (tomada de Alfaro de Lanzone 1985: 571). Derecha: estado actual.

anteriores sobre el tema." (González y Díaz 1992:19). Otro aspecto a resaltar es que "Careció al parecer [...] de ventanas u otras aberturas. De acuerdo a los datos recogidos, todo hace pensar que las cuatro paredes tuvieron una altura uniforme, lo que lleva a concluir que la C.M. responde al tipo más común de arquitectura doméstica incaica, y que por lo tanto su techado debió tener caída a dos aguas, con hastiales de adobe o más probablemente de paja." (González y Díaz 1992:20). Además, la "...C.M. y las estructuras circundantes se sitúan en una especie de terraza o plataforma natural sobreelevada con respecto del resto de la población situada al este y al Sur." (1992:20).

Respecto de la Casa Morada como estructura intrusiva en un sector de estructuras locales, quiero resaltar dos observaciones realizadas por estos investigadores. En primer lugar, señalaron que difícilmente la estructura en la que se emplazó Casa Morada y la estructura inmediatamente al Norte de ella conserven su forma original toda vez que "En general, las plantas de todas las estructuras de población tienen una cierta regularidad con predominio rectangular..." (1992:21). Adicionalmente, señalan que el muro Este de la estructura número 6 (noroeste de la estructura número 3), que alcanzaba 1,4 $\mathrm{m}$ de altura al momento del relevamiento, debió haber sido parcial o totalmente construido por los Inkas dado que es muy "parejo" y presenta bloques de arenisca roja al igual que Casa Morada. En un evento constructivo posterior, se clausuró la puerta de vano rectangular (0.92 - 0,98 m según González y Díaz 1992: 22) que se había construido en su sección septentrional. En su sección meridional, destacan un acceso que comunica las estructuras 6 y 3 con una extensión o "diente" (1992: 22) no observable en la actualidad y cuya factura podría apoyar esta hipótesis (Figura 6). Otro tanto puede señalarse respecto de las estructuras de al- macenaje subcuadrangulares detectadas al Sur de la estructura número 6 , cuya morfología lleva a los autores a señalar que "seguramente se trata de collcas construídas en la misma época en la que se construyó Casa Morada y que pertenecían al mismo propietario de ésta" (González y Díaz 1992: 44). Inferir la cronología de los eventos constructivos que llevaron a aquella sección clausurada o reconstruida amerita otra clase de investigación y no será un tema a tratar aquí. Sí corresponde destacar que la posibilidad de que hayan existido intervenciones adicionales a la intrusión de Casa Morada ha sido considerada previamente. Sin embargo, aquellas intervenciones no se han considerado a la luz del diagrama general de articulación de los diferentes sectores que conforman el asentamiento.

En los trabajos de prospección y relevamiento, se ha evaluado del sector 13 en su conjunto: 1. La posibilidad y condiciones de acceso al sector, 2. La articulación interna de las estructuras, es decir las posibilidades de circular en función de las técnicas constructivas empleadas y los accesos identificados, y 3 . La accesibilidad física a Casa Morada en particular.

\section{Accesibilidad visual y física al Sector 13}

El hecho de que este sector se encuentre en la superficie más elevada del terreno en el que se emplaza el sitio, crea ciertas condiciones de base para su accesibilidad visual (Figura 7). Se presenta cada una de ellas, desde cada uno de los puntos cardinales y su accesibilidad física en función de las vías de circulación que confluyen en el sector.

Accesibilidad visual y fisica oriental. La accesibilidad visual 


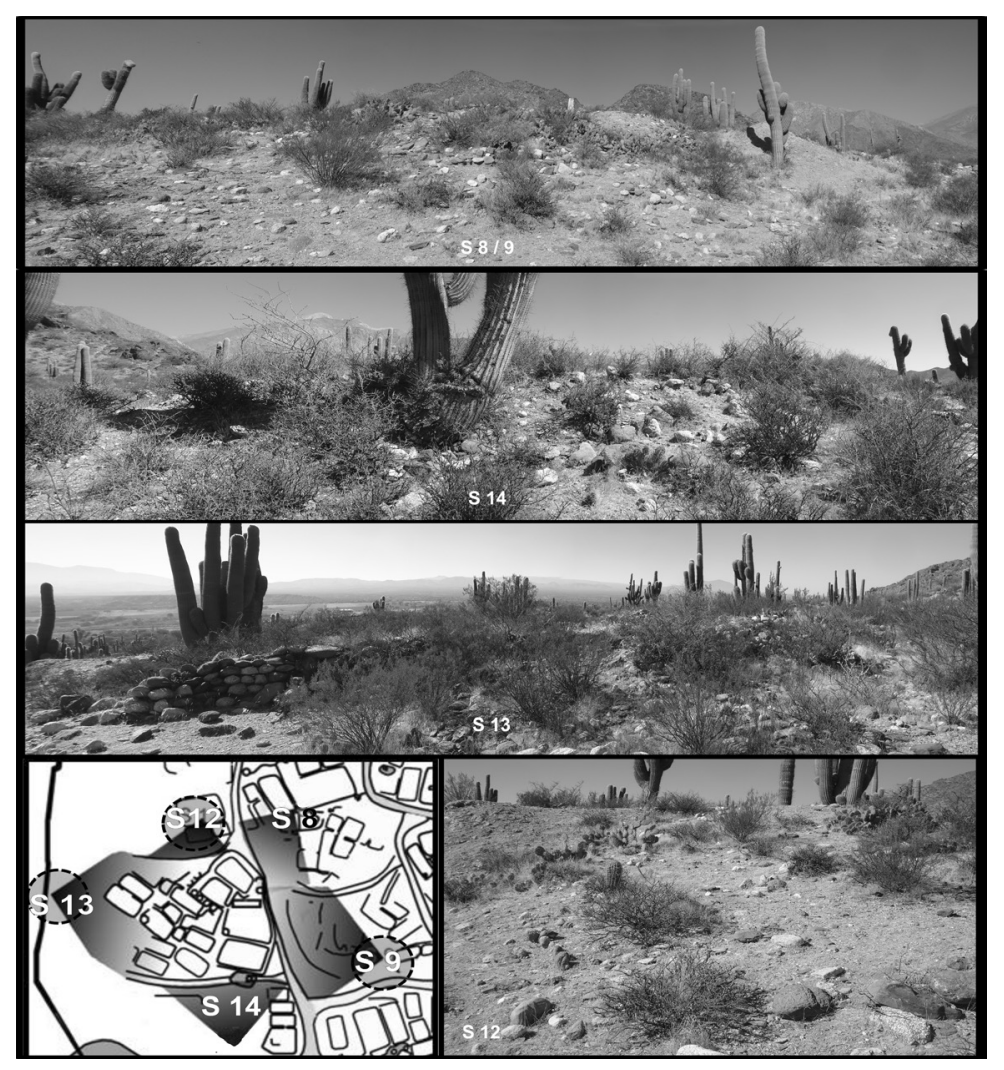

Figura 7: Accesibilidad visual al Sector 13 desde los sectores adjuntos.

desde el Este (Figura 7) está impedida dada la marcada inclinación de la pendiente hacia los sectores 8 y $9\left(35^{\circ}\right.$ $25^{\circ}$ de inclinación a $6 \mathrm{~m}$ respectivamente $)^{6}$. Esto implica que desde aquellos sectores locales no podrían visualizarse las estructuras del Sector 13. Esto sí ocurre cuando se recorre la vía de circulación principal número 1, pero debe aclararse que lo que podría visualizarse permanentemente son los espacios inter edilicios, la superficie interna de las estructuras inmediatamente adjuntas $\left(\mathrm{n}^{\circ} 2\right.$ y 3) y, por supuesto, el exterior de Casa Morada. No así las superficies internas de las estructuras restantes, que en perspectiva se esconden tras los muros. Respecto de la accesibilidad física al sector, a la altura en que la vía principal de circulación se aproxima hacia el acceso a la estructura $\mathrm{n}^{\circ} 3$, su ancho medio $(2,71 \mathrm{~m})$ se reduce a o, 6 $\mathrm{m}$. Si bien esta disminución no es abrupta sino gradual, el número de personas que podía circular por ella a esa altura se encontraba permanentemente restringido (Figura 8).

Accesibilidad visual y fisica meridional. La posibilidad de ac- ceder visualmente desde el sector inmediatamente al Sur (14), se ve impedida por la vía de circulación secundaria que se eleva $2 \mathrm{~m}$ respecto de él (Figura 7) y que lo separa del sector 13. Sin embargo, presenta la particularidad de que la superficie transitable se encuentra a nivel de los espacios interedilicios del Sector 13, de modo que transitarla permite la visualización de las superficies internas de aquellas estructuras que limitan con ella. Por supuesto, la accesibilidad visual se limita al interior de la estructura 2, parcialmente hacia la estructura 3, y al exterior de Casa Morada. Respecto de la accesibilidad física al sector,

6 Debo mencionar que González y Díaz (1992: 29) consideraron que debía evaluarse seriamente la posibilidad de que a lo largo de la pendiente hacia lo que aquí he señalado como la vía de circulaciónsecundaria que divide los sectores 8 y 9 , existiese una forma de rampa al sector que contiene Casa Morada. No he observado algo que apoye esta posibilidad, pero no debe descartarse. 


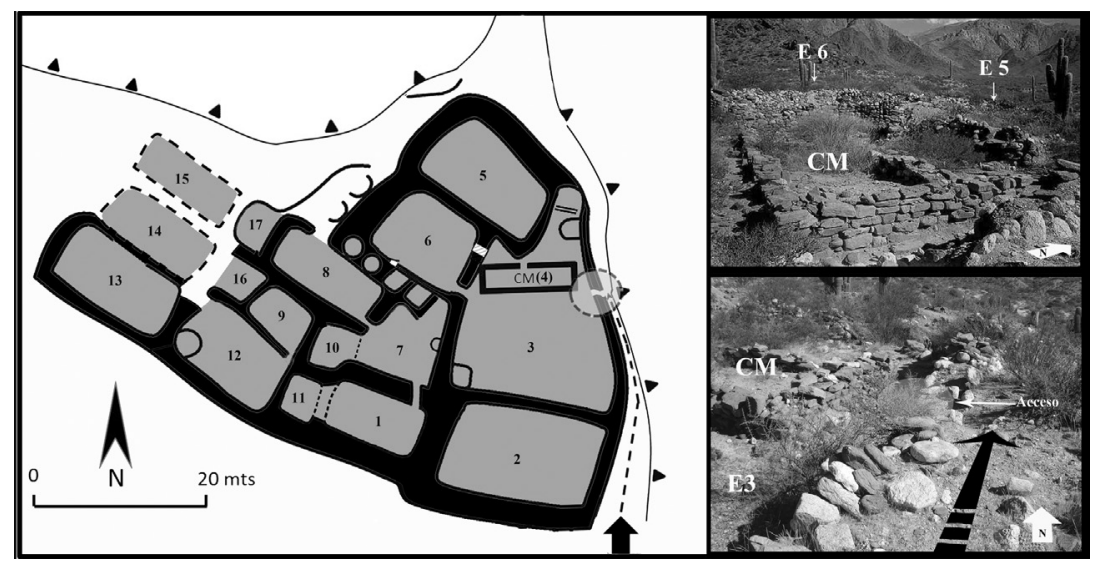

Figura 8: Accesibilidad física y visual desde vía de circulación principal.

debió implicar redirigirse primero hacia la vía principal de circulación principal en donde se encuentra el acceso a la estructura n 3 (Figura 8).

Accesibilidad visual y fisica occidental. Las condiciones de accesibilidad desde el Oeste son las más difíciles de establecer. No resulta claro aún si aquella zona en la que se han documentado tan pocas estructuras, ha sufrido procesos modernos de destrucción y quita selectiva de rocas. Sin embargo, la ausencia de estructuras observables e incluso ángulos de contacto dispersos, contrasta notablemente con la presencia del complejo de estructuras en que se encuentra Casa Morada, destacando que no existe pendiente abrupta desde el lado Oeste. El único límite consiste en que el lado externo de los muros occidentales de las estructuras 5; 6 y 13 se encuentran elevados actualmente hasta $0,5 \mathrm{~m}$ respecto de la superficie actual. Esto me lleva a inferir una accesibilidad visual restringida a la cara externa de los muros y una accesibilidad física restringida a la periferia del complejo de estructuras. (Figura 9).

Accesibilidad visual y fisica septentrional. Al igual que en los anteriores casos, la accesibilidad visual desde el sector adjunto (12) se ve impedida por la diferencia de nivel. Sin embargo, existe una particularidad a destacar respecto de la accesibilidad física: la presencia de una vía de circulación secundaria que separa los sectores 8 y 12 , confluye con la vía de circulación principal $n^{\circ} 2$ que desemboca en la entrada al sitio usualmente señalada, y desemboca directamente en el perímetro del sector 13. Ahora bien,

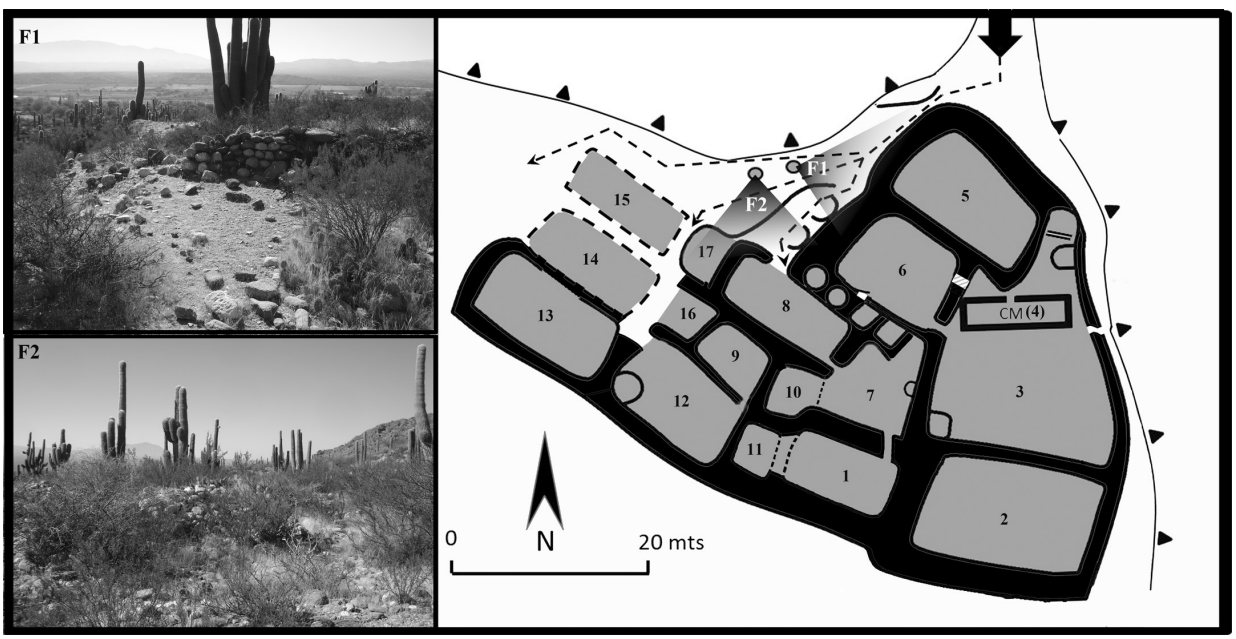

Figura 9: Accesibilidad física y visual desde vía de circulación secundaria hacia el Oeste.

$64 \mid \begin{aligned} & \mathrm{N}^{\circ} 53 / 2016 \\ & \text { ESTUdios ATACAMEÑos } \\ & \text { Arqueología y Antropología Surandinas }\end{aligned}$ 


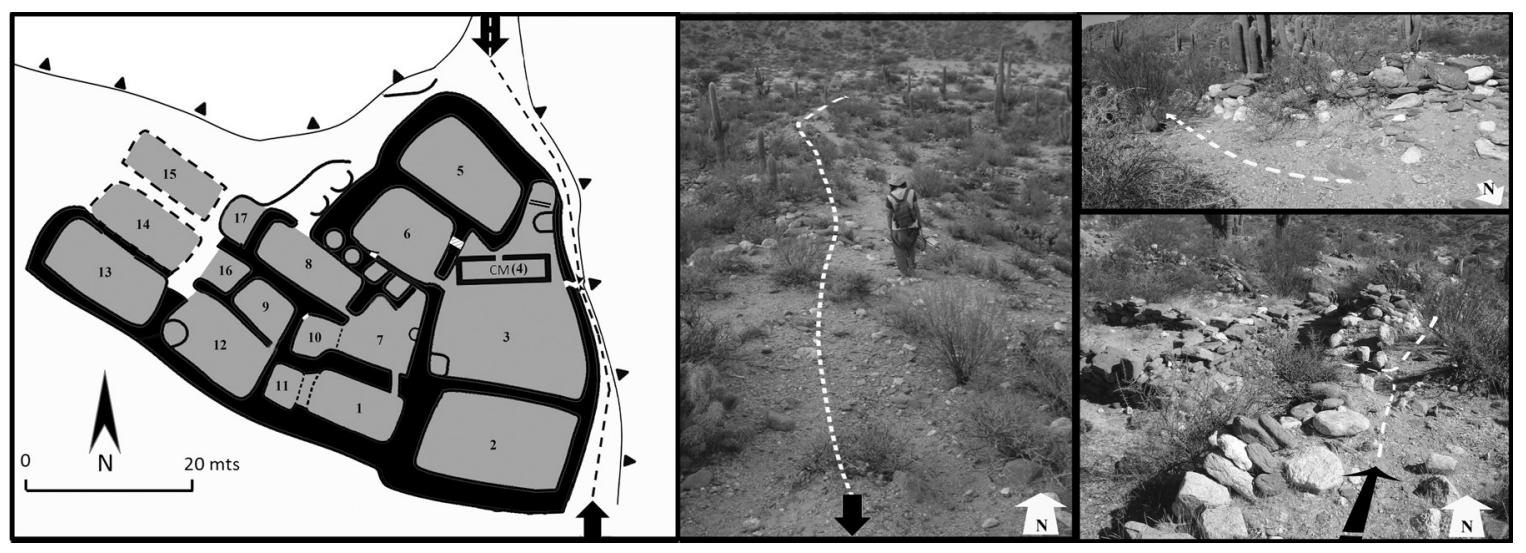

Figura 10: Accesibilidad física y visual desde vía de circulación secundaria hacia el Este y confluencia con vía de circulación principal.

esta desembocadura presenta una particularidad; no bien accede al perímetro del sector, se desdobla en dos direcciones. Hacia el Oeste, encuentra un muro de contención hacia el sector 12 y tan sólo a 0,4 $\mathrm{m}$ del vértice exterior Noroeste de la estructura $n^{\circ} 5$. Trascender este estrecho "acceso" sólo habilitaba la circulación hacia la periferia del sector, tras los muros de las estructuras 5 y 6 (Figura $n^{\circ}$ 9). Hacia el Este, se conecta con la vía principal de circulación $n^{\circ} 1$ cuyo estrechamiento ya fue señalado (Figura 10).

De la exposición precedente, derivo que la accesibilidad visual y física hacia el sector en donde se emplazó el complejo de estructuras que aloja a Casa Morada se encuentra restringida, desde lo físico, por la cantidad de gente que podía transitar las vías de circulación que lo rodean. Desde lo visual, por su posición topográfica respecto de los sectores adjuntos. Adicionalmente, no puede afirmarse que circular por aquella zona haya sido condición necesaria para arribar a otro sector en el sitio. Cualquier sector estrictamente local es fácilmente accesible a través de la articulación de vías de circulación principales y secundarias sin obstáculos o estrechamientos como los documentados aquí.

\section{ARTiCULACIÓn INTRASECTORIAL}

Las técnicas constructivas empleadas y el diseño de planta de las estructuras presentes en el sector 13, exceptuando Casa Morada y las intervenciones ya mencionadas, no parecen diferir de las señaladas anteriormente para la arquitectura local. Es decir, se trata de estructuras construidas con rodados locales, predominando las estructuras de planta subrectangular y las restantes estructuras son subcuadrangulares, circulares y semicirculares internas, y la gran mayoría de los ángulos de contacto entre los muros son curvos, exceptuando aquellos que son el producto de agregados posteriores a un trazado perimetral.

Sin embargo, existen una serie de diferencias fundamentales respecto de los conjuntos estrictamente locales. Específicamente, el ancho de los muros y por consiguiente de los espacios interedilicios, las técnicas constructivas de estos últimos (presencia/ausencia de rocas en su superficie), y la presencia / ausencia de accesos a las estructuras y su distribución.

Se mencionó anteriormente que las estructuras al interior de los sectores estrictamente locales, los espacios interedilicios oscilan mayoritariamente entre los o,6 y 1,2 m. Claramente, no es el caso de las estructuras que rodean y alojan a Casa Morada (Tabla 1). En primer lugar, el ancho de los espacios interedilicios oscila entre los $0,91 \mathrm{~m}$ (estructura $\mathrm{n}^{\circ} 12$, espacio interedilicio Este; promediados con González y Díaz 1992) y los 2,7 m (estructura $\mathrm{n}^{\circ}$ 1, espacio interedilicio Sur; 2,85 $\mathrm{m}$ promediados con González y Díaz 1992). Si tomamos el promedio de los espacios interedilicios que rodean a cada estructura, aquellos se ubican en el intervalo de los 0,9 m (Estructura 11) a los 2,29 m (Estructura 2), ubicándose la mayoría de los anchos promedio entre 1,25 y 2,02 $\mathrm{m}$ (Estructuras $1 ; 3 ; 5 ; 6 ; 7 ; 8 ; 9 ; 10 ; 12$ ). 


\begin{tabular}{c|c|c|c|c|c|} 
Estructura & Sur & Este & Norte & Oeste & EI $\bar{X}$ \\
\hline 1 & 2,85 & 2,4 & 1,7 & 0,9 & 1,96 \\
\hline 2 & 2 & 2,34 & 2,4 & 2,4 & 2,29 \\
\hline 3 & 2,4 & 1,87 & 1,1 & 1,675 & 1,76 \\
\hline 5 & 1,7 & 1,48 & 2,6 & 2,3 & 2,02 \\
\hline 6 & 3,5 & 0,95 & 1,7 & 2 & 2,04 \\
\hline 7 & 1,7 & 1,67 & 1,3 & 1,6 & 1,57 \\
\hline 8 & 2 & 1,6 & 3,5 & 1,25 & 2,09 \\
\hline 9 & 1,55 & 1,27 & 2 & 1,15 & 1,49 \\
\hline 10 & 1,2 & $-\cdots--$ & 1,4 & 1,37 & 1,32 \\
\hline 11 & 1,55 & 0,57 & 0,55 & 0,91 & 0,90 \\
\hline 12 & 1,24 & 0,91 & 1,6 & 1,25 & 1,25 \\
\hline
\end{tabular}

Tabla 1: Relevamiento del ancho de los espacios interedilicios de las estructuras que componen el complejo que aloja a Casa Morada. Atendiendo a la incidencia de procesos de formación que puedan haber afectado la integridad de los muros, se promediaron los registros de González y Díaz 1992 (cuando presentes) con los actuales (celdas grises).

En segundo lugar, varios espacios interedilicios presentan rocas encastradas de modo vertical u oblicuo en toda su superficie (Figura 11). Cuando aquello ocurre en sectores locales, se da únicamente en los muros divisorios transversales de ciertas estructuras subrectangulares y representan tan solo el 7,2\% de los recintos presentes.

En tercer lugar, y en línea con lo expuesto hasta aquí, llama la atención la articulación entre las estructuras, ya que los tipos de accesos identificados exhiben una frecuencia y una distribución que contrasta con el modo local de cir- cular y acceder predominantemente interedilicio. Aclaro que sólo se consideraron aquellas estructuras que poseen los cuatro muros perimetrales bien conservados. A saber, sólo dos estructuras carecen de accesos claramente identificables $\left(n^{\circ} 5\right)$ o bien resultan poco confiables $\left(n^{\circ} 2\right)$. Las restantes 10 estructuras aquí consideradas por motivos de preservación, poseen mayoritariamente accesos directos desde otras estructuras, secundados por accesos desde espacios interedilicios con rampa (Figura 12), y por un acceso desde la vía de circulación principal número 1. El tratamiento de los anchos de los accesos es un aspecto

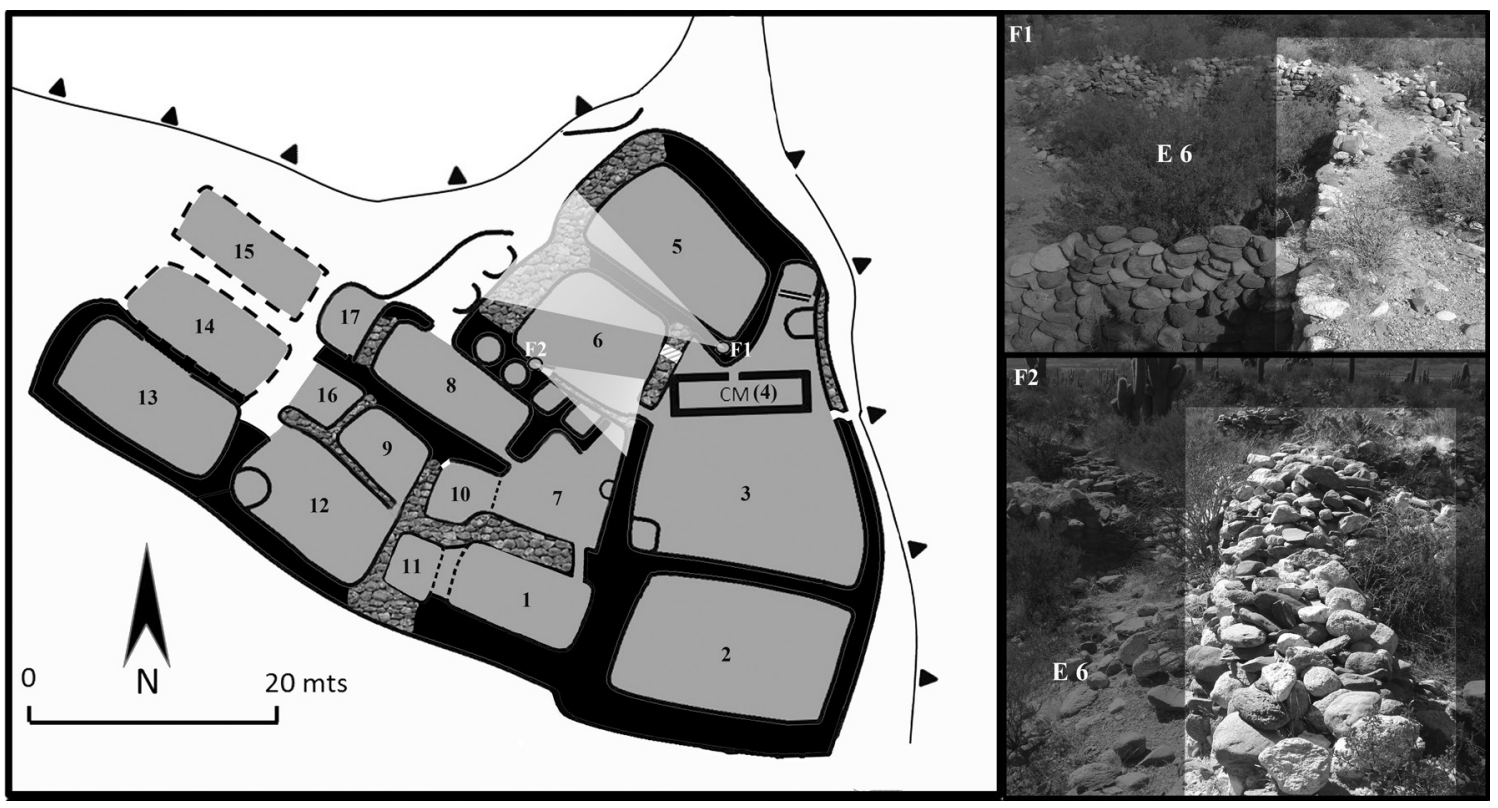

Figura 11: Ejemplo de espacio interedilicio despejado $\left(\mathrm{F}_{1}\right)$ y bloqueado $\left(\mathrm{F}_{2}\right)$. 


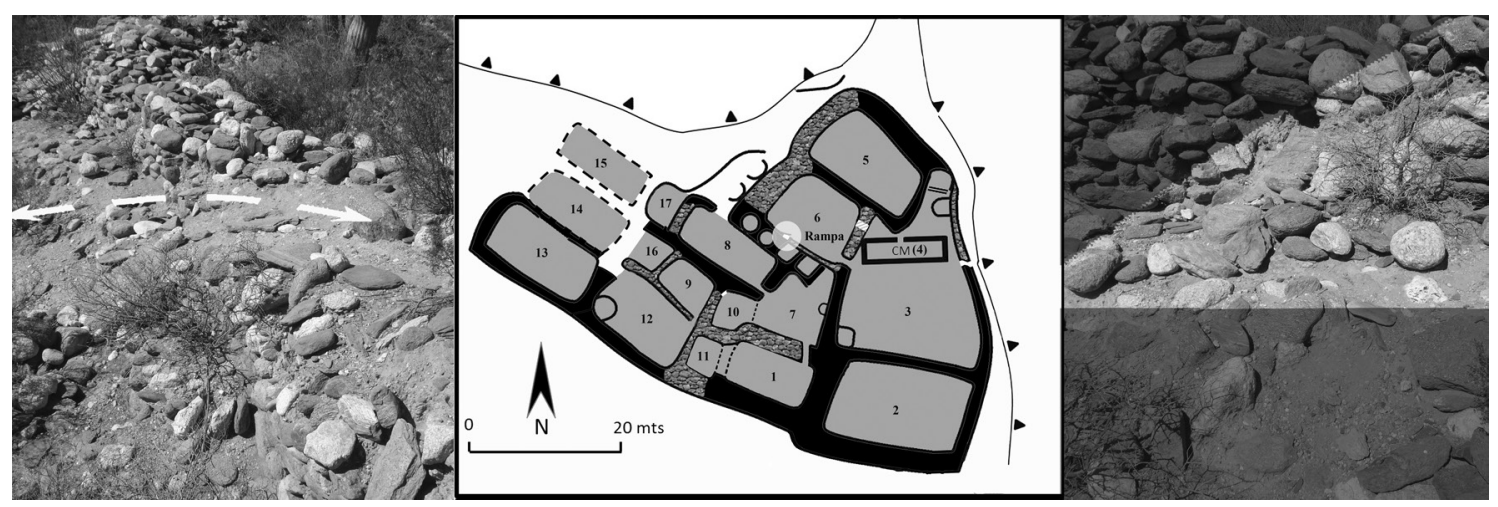

Figura 12: Ejemplo de rampa (hacia recinto nº 6).

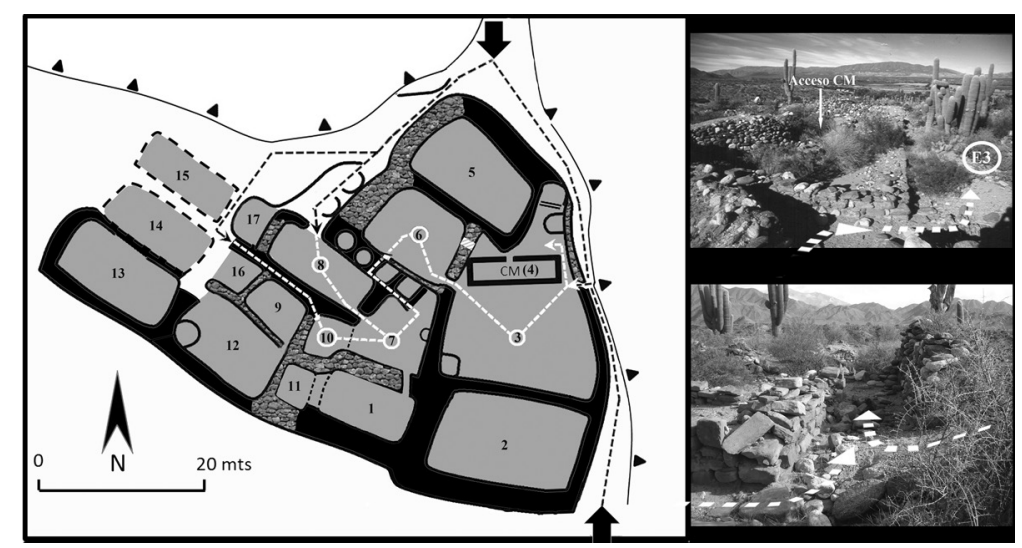

Figura 13: Potenciales rutas internas al complejo de estructuras que alberga a Casa Morada.

que quedará pendiente (ver Figura 6). La conservación actual impide determinar sus límites con seguridad y menos aún utilizarlos como parámetro comparativo para establecer diferencias con los accesos en sectores estrictamente locales.

En definitiva, el diagrama general de articulación y circulación al interior del sector 13 , encontraba instancias muy pautadas y reguladas. La presencia de espacios interedilicios bloqueados canalizaba la circulación interedilicia coordinando y regulando la movilidad al interior del complejo de estructuras. Finalmente, como soporte al control de la circulación interedilicia, la frecuencia relativa de accesos directos garantizaba que la circulación se diese predominantemente de estructura en estructura.

\section{Accesibilidad física a Casa Morada}

La distribución de accesos y espacios interedilicios descripta más arriba crea ciertas condiciones de accesibilidad física a Casa Morada, estableciendo tres rutas potenciales en el interior del complejo (Figura 13).

Independientemente de la que se considere, los trayectos señalados garantizaban que la posibilidad de acceder físicamente a Casa Morada implicaba necesariamente transitar previamente otras estructuras y jamás estar habilitado para observar lo que sucedía en su interior. Todas las rutas físicas hacia el interior de Casa Morada debieron implicar previamente acceder a la estructura número tres y rodear Casa Morada por el lado contrario a su acceso, 
para eventualmente transitar el pequeño espacio entre muros hacia el Este de ella.

En conjunto, este diseño general de plantas, espacios interedilicios y accesos, expresa una intencionalidad manifiesta en la habilitación y censura de ciertas rutas y, junto con la accesibilidad visual y física al sector, la inhibición de la participación de lo que allí sucediese para aquellos segmentos que habitaban los sectores estrictamente locales.

\section{Discusión: La Intervención Inka en La Paya. Escenarios Múltiples e Hipótesis Plausibles}

Tres tendencias fundamentales caracterizaban al modo de habitar local: 1. La homogeneidad y redundancia edilicia en lo que concierne a las técnicas constructivas aplicadas, los tipos edilicios presentes en los sectores, y la regularidad compositiva de ellos, 2. La alta articulación tanto entre sectores como a su interior, y 3 . La permeabilidad visual del interior de los sectores y posiblemente al interior de sus estructuras no techadas. Estas tres condiciones de base, en el ejercicio de recorrer La Paya, exponían a los sujetos a un paisaje de repeticiones que no buscaba restringir las posibilidades ambulatorias y evitar el conocimiento de las actividades que desarrollaban otros. Por el contrario, recorrer los senderos elevados de La Paya y habitar sus estructuras implicaba necesariamente ponerse en contacto con otras personas y sus performatividades. Esto complementa investigaciones previas que señalaron la ausencia de evidencias concretas de diferenciación en las actividades llevadas a cabo en las estructuras que componían uno de los sectores de La Paya (Díaz 1981) y en otros conglomerados locales (ver en Díaz 1981; Acuto 2007; Acuto et al. 2008).

Consideraciones previas sobre la arquitectura de este complejo adjudican la intervención inkaica a: Casa Mora$\mathrm{da}$, la estructura $\mathrm{n}^{\circ} 3$ y 5 , la presencia de un muro divisor y la posterior clausura de un acceso en su sección septentrional, las estructuras de almacenaje, y dos estructuras semicirculares internas al recinto 3. He expuesto aquí que la intervención debe extenderse hacia la totalidad del complejo de estructuras, comenzando por la restricción a su accesibilidad mediante la intervención de las vías de circulación principal y secundaria que desembocan en el sector 13 .
Cabe preguntarse en esta instancia si las características de este complejo arquitectónico son exclusivamente producto de transformaciones impulsadas por el Tawantinsuyu, o bien preceden al arribo imperial. En este último escenario, el complejo arquitectónico que alberga a Casa Morada habría sido precedente y albergado a un grupo de jerarquía o al menos espacialmente diferenciado respecto del resto de la población. Con este grupo, los representantes imperiales habrían incentivado y mantenido una relación más estrecha, lo que se reflejó en el emplazamiento de Casa Morada en su complejo y en la adquisición y utilización de bienes foráneos. Incluso sería posible agregar a este constructo hipotético que la circulación mayoritariamente sobreelevada promovida por las vías de circulación principales y secundarias reflejaba cierta intencionalidad imperial por controlar las actividades que se llevaran a cabo al interior de los otros sectores locales. Otro tanto puede señalarse respecto de la distribución de las vías de circulación. Después de todo, incentivar y/o garantizar la circulación y la permeabilidad física bien podría estar orientada a asegurar el control del resto de la población (Nielsen 1995: 57)

Sin embargo, la plausibilidad de este escenario hipotético debe ponderarse a la luz de otras evidencias que han arrojado previas investigaciones en la región.

En primer lugar, los relevamientos realizados en otros sitios de la región exhiben configuraciones similares a lo expuesto aquí para La Paya. Esto es, una sectorización mediante senderos sobreelevados que circunvalan conjuntos de estructuras con similares técnicas constructivas, diseños de planta, y una uniformidad relativa en su composición edilicia. Esto indica, por un lado, que la configuración general por sectores bien puede preceder al arribo imperial. Por otro, que cierta homogeneidad edilicia es una característica recurrente al menos en esta región.

Asimismo, esperaría que la existencia de este grupo se refleje en otras esferas de la vida social de estas comunidades. Sin embargo, las investigaciones relativas a los entierros de La Paya han mostrado una baja diferenciación respecto de las técnicas constructivas de las cistas y los materiales depositados en su interior. Esto es, las prácticas mortuorias en general no persiguieron la distinción y/o exaltación de individuos o grupos. Por el contrario, 
aquellas no presentan diferencias estadísticamente significativas respecto de los materiales depositados, remiten mayoritariamente a esferas de la vida cotidiana de las personas, no exhiben producción exclusiva para el acompañamiento de los difuntos, y presentan una frecuencia relativa de materiales foráneos y/o ligados a esferas rituales extremadamente baja (ver en Acuto et al. 2014).

Finalmente, las excavaciones que se realizaron previamente en la estructura $\mathrm{n}^{\circ} 6$ (ver Alfaro de Lanzone 1985: 574-577) revelaron que la mayoría del material cerámico es de origen e influencia Inka sin claras evidencias de ocupaciones precedentes.

\section{Conclusiones}

La dualidad del espacio socialmente producido lo involucra inevitablemente en luchas por su reproducción, destinadas a la manutención de la espacialidad existente o a la transformación de ciertos aspectos de ella, lo que habilita la coexistencia de espacialidades "de" o "para" la diferenciación. Entre ellas, se encuentra la modificación de las condiciones de posibilidad para las interacciones interpersonales y el acceso a diferentes tipos de recursos.

En La Paya, la intervención imperial parece haber cooptado un aspecto espacial y organizacional local (la sectorización) para adecuarlo a la nueva realidad imperante. Allí en donde acceder a un sector implicaba caminar previamente por senderos sobreelevados, se impuso una restricción de base cuyo soporte topográfico y arquitectónico implicó limitar la cantidad de personas que podían transitar por allí e inhibir la visualización del sector. Allí en donde recorrer los senderos locales implicaba experiencias frente a las actividades que realizaban otros miembros de la comunidad, se impuso un carácter restrictivo reservado sólo a aquellos que efectivamente podían bordear y acceder al complejo de estructuras del sector 13, y una imposibilidad visual y por lo tanto comunicativa respecto de los habitantes de los sectores restantes. La participación estrictamente local de las actividades que allí tenían lugar, fue anulada. Esta dimensión de diferenciación debe de haber creado la primera fisura en los segmentos locales y creado la primera distinción: entre los habitantes del sector 13 y aquellos que residían en los sectores estrictamente locales.
Ahora bien, ¿qué implicaba la organización interna del sector 13 para sus habitantes?

Residir en el complejo de estructuras en el que se encuentra Casa Morada trastocó el modo local de habitar insertando nuevas condiciones de posibilidad para ver, caminar, y participar de las actividades que se realizaban tanto en el resto del sitio como en las estructuras contiguas. Se introdujo una nueva forma de habitar e interactuar que se fundó en una restricción a la experiencia ambulatoria e implicó una circunscripción general de los saberes ambulatorios previamente interiorizados.

Aquí es donde Casa Morada interviene de un modo sustancial como segunda dimensión de ruptura con los saberes ambulatorios locales. La presencia de subdivisiones al interior de las estructuras locales se reducía a un muro divisor que habilitaba la conexión entre los espacios resultantes, tal como podría ser el caso de las estructuras 6 y 3 . Aquí se agregó una dimensión adicional de subdivisión que implicó emplazar una estructura al interior de un perímetro de modo que aseguraba la imposibilidad permanente de ver el interior de Casa Morada. Aún en el caso de efectivamente trascender aquel pasillo $(1-1,45$ m en González y Díaz 1992:23) en el que confluyen todas las rutas, ver hacia el interior de Casa Morada hubiese sido imposible ya que para ello se debía estar directamente en frente del acceso. Aun considerando la posibilidad de circular por los espacios interedilicios al Sur y al Este de la estructura número 5 , los datos estratigráficos de la estructura $n^{\circ} 6$ presentados por Alfaro de Lanzone (1985) permiten proyectar la altura de los muros de las estructuras que rodeaban a Casa Morada dentro del rango de los $2 \mathrm{~m}$. Aun proyectando una altura potencial para el acceso de Casa Morada de 1,7 m, ver hacia el interior hubiese estado notablemente restringido para quien/es circulasen por aquellos espacios. La clausura del acceso en la sección septentrional del muro oriental de la estructura n 6 y su relocalización al Sur, refuerza la privacidad de Casa Morada al redireccionar la circulación por atrás del muro contrario a donde se localizó su acceso.

Esto constituyó la segunda fractura en el segmento local, adicional a aquella que señalé anteriormente y ahora interna al sector 13; entre quien o quienes habitasen Casa Morada y quienes habitasen las estructuras que la rodean. 
Se ha planteado que una de las estrategias imperiales puestas en práctica en el Valle Calchaquí Norte consistió en crear nuevos paisajes y lugares que reeducaran y resocializaran a las poblaciones locales (Acuto 1999). Esto quizás resulte más notorio en aquellos sitios predominantemente Inka, o bien en aquellos que presenten espacios para la participación coordinada de segmentos de las poblaciones locales en prácticas y eventos diagramados por el imperio. Tal puede ser el caso de las plazas de Guitián, inmediatamente en frente de La Paya, y el complejo de Cortaderas Bajo (ver en Acuto y Gifford 2007; Acuto 2010; Acuto et al. 2012). La Paya carece aparentemente de aquellos espacios; con seguridad, carece de espacios formalizados de una envergadura y factura comparable a los anteriormente mencionados.

Sin embargo la localización topográfica del sector 13 y su reorganización arquitectónica, introdujo una experiencia del habitar que lo trascendía y, en la cotidianeidad, socavaba la espacialidad local para insertar al menos dos dimensiones experienciales de la diferencia: entre los habitantes de las estructuras que rodeaban a Casa Morada y aquel o aquellos que residían en ella, y entre los habitantes del sector 13 y aquellos de los sectores estrictamente locales. En el primer caso, la experiencia de la diferencia operaba a escala del cuerpo, nutriéndose de una transformación de los saberes ambulatorios previamente interiorizados. En el segundo caso no se trataba de una dimensión experiencial estrictamente ambulatoria que operase a escala del cuerpo, sino una predominantemente comunicativa que subvertía las condiciones de interacción interpersonal que caracterizaban al habitar local. Ambas creaban nuevas condiciones de interacción, demarcaban el contraste entre colonizadores y colonizados, y creaban condiciones espaciales y materiales para la cristalización de nuevos segmentos dentro de la comunidad local.

\section{Agradecimientos}

Este trabajo fue realizado con el apoyo de Heinz Grant for Latin American Archaeology y Wenner-Gren Foundation for Anthropological Research, International Collaborative Research Grant. Agradezco a la comunidad Diaguita -Kallchaki por interesarse y participar activamente en discusiones y trabajos de campo, y por la hospitalidad y predisposición que han mostrado a lo largo de los años. Agradezco especialmente a Félix Acuto e Iván Leibowicz por la lectura y las contribuciones realizadas a este manuscrito. Finalmente, a los editores y a los evaluadores quienes, desde el anonimato, sin duda han mejorado este artículo con sus observaciones. 


\section{$\bullet$ Referencias citadas}

ACUTO, F. 1999. Paisaje y dominación: la constitución del espacio social en el imperio Inka. En Sed non satiata. Teoría social en la arqueología latinoamericana contemporánea. A. Zarankin y F. Acuto (Eds.), pp. 33-75. Ediciones Del Tridente, Buenos Aires, Argentina.

ACUTO, F. 2007. Fragmentación versus integración comunal: Repensando el Período Tardío del Noroeste Argentino. Estudios Atacameños 34: 71-95.

ACUTO, F. 2010. Living under the imperial thumb in the Northern Calchaquí Valley (Argentina). En Distant provinces in the Inka empire: Toward a deeper understanding of Inka imperialism, M. Malpass y S. Alconini (Eds.), pp. 108-150. University of Iowa Press, Iowa City.

ACUTO, F. y C. GIFFORD. 2007. Lugar, arquitectura y narrativas de poder. Relaciones sociales y experiencia en los centros Inkas del Calchaquí Norte. Arqueología Sudamericana 3 (2): 135-161.

ACUTO, F.; C. AMUEDO; M. KERGARAVAT; A. FERRARI; L. GAMARRAyA. L. GOLDIN. 2008. Experiencias subjetivas en las aldeas prehispánicas del valle Calchaquí Norte:Arqueología de la vida cotidiana, prácticas y relaciones sociales durante el Periodo Prehispánico Tardío. En Arqueología del extremo sur del continenteamericano. Resultados de nuevos proyectos, L.A. Borrero y N. Franco (Eds), pp. 11-54, CONICET - IMHCIHU. Buenos Aires.

ACUTO, F. A.; M. KERGARAVAT y C. AMUEDO. 2011. Experiencia de la muerte y la representación de las personas en las prácticas funerarias del valle Calchaquí Norte. Comechingonia, Revista de Antropología 14: 23-54.

ACUTO, F.; M. KERGARAVAT, y C. AMUEDO. 2014. Death, personhood, and relatedness in the South Andes a thousand years ago. Journal of Material Culture 19:303-326.

ACUTO, F.; A. TRONCOSO y A. FERRARI 2012. Recognising strategies for conquered territories: a case study from the Inka North Calchaquí Valley. Antiquity 86: 1141 - 1154.

ALCONINI, S. 2010. Yampara households and communal evolution in the southeastern Inka peripheries. En Distant provinces in the Inka empire: Toward a deeper understanding of Inka imperialism, M. Malpass y S. Alconini (Eds.), pp. 75-107. University of Iowa Press, Iowa City.

ALFARO DE LANZONE, L. 1985. Investigación arqueológica de la 'ciudad' prehistórica de La Paya, Dpto. Cachi, provincia de Salta, Argentina. Beiträge zur allgemeinen und vergleichenden archäeologie 7: 563-595.
AMBROSETTI, J. B. 1902. El sepulcro de La Paya recientemente descubierto en los Valles Calchaquíes (Provincia de Salta). Anales del Museo Nacional de Buenos Aires, Tomo VIII (Ser. $\left.3^{\mathrm{a}}, \mathrm{t} / \mathrm{1}\right)$ : 119148. Buenos Aires, Argentina.

AMBROSETTI, J. B. 1907. Exploraciones arqueológicas en la ciudad prehistórica de La Paya (Valle Calchaquí, Pcia. de Salta). Revista de la Universidad de Buenos Aires Tomo VIII; Sección Antropología. Facultad de Filosofía y Letras, Buenos Aires.

AMUEDO, C. 2010. La muerte de niños y su tejido de materialidad. Prácticas, representaciones y categorías construidas en las tumbas de infantes en vasijas, Periodo Tardio (900-1470 DC), valle Calchaquí Norte. Tesis de Licenciatura, Facultad de Filosofía y Letras, Universidad de Buenos Aires.

BALDINI, L. 1980. Dispersión y cronología de las urnas de tres cinturas en el Noroeste argentino. Relaciones XIV (1): pp. 4961.

BALDINI, L. y G. BALBARREY. 2004. Análisis de pastas cerámicas tardías del valle Calchaquí central (Salta, Argentina). Chungará, Vol. 1.Tomo II: $1069-1080$.

BALDINI, L. y M. SPROVIERI. 2009. Vasijas negras pulidas. Una variedad de la cerámica tardía del valle Calchaquí. Estudios Atacameños 38: 21-38.

BELL, C. 1992. Ritual theory, ritual practice. Oxford University Press, New York.

BENNETT, W.; BLEILER, E. y F. SOMMER, 1948. Northwest argentine archaeology. Yale University Publications in Anthropology, N 38, New Haven.

BOMAN, E. 1908. Antigüedades de la región andina de la República Argentina y el desierto de Atacama. Tomos 1 y 2 . Universidad Nacional de Jujuy. Argentina.

BOURDIEU, P. 1977. Outline of a theory of practice. Cambridge University Press, Cambridge.

BOURDIEU, P. 2007. El sentido práctico. Siglo Veintiuno Editores, Buenos Aires.

BUDDEN, S. y J. SOFAER. 2009. Non-discursive knowledge and the construction of identity: Potters, potting and performance at the Bronze Age Tell of Százhalombalta, Hungary. Cambridge Archaeological Journal 19 (2): pp. 203-220. 
BURGUER, R. L.; C. MORRIS y R. MATOS MENDIETA. 2007. Variations in the expression of Inka power. Dumbarton Oaks Research Library and Collection, Washington D.C. Harvard University Press.

CALDERARI, M, 1991. Estilos cerámicos incaicos de La Paya.Actas del XI Congreso Nacional de Arqueología Chilena, Tomo II: 151-164. Museo Nacional de Historia Natural, Sociedad Chilena de Arqueología, Santiago de Chile.

CALDERARI, M. y V. WILLIAMS. 1991. Re-evaluación de los estilos cerámicos incaicos en el Noroeste Argentino. Comechingonia 9 (2): 75-96.

CERUTI, M. C. 2003. Llullaillaco: Sacrificios y ofrendas en un santuario Inca de alta montaña. Publicación del Instituto de Investigaciones de Alta Montaña. Ediciones de la Universidad Católica de Salta, Salta.

COVEY, A. 2015. Inka Imperial Intentions and Archaeological Realities in the Peruvian Highlands. En The Inka Empire. AMultidisciplinary Approach, I. Shimada (Ed), pp. 83-95. William and Bettye Nowlin Series in Art, History, and Culture. Texas University Press.

CSORDAS, T. 1990. Embodiment as a paradigm for anthropology. Ethos, Vol. 18 (1): 5-47.

D'ALTROY, T. N., 1992. Provincial power in the Inka Empire. Smithsonian Institution Press, Washington.

D'ALTROY, T. N. 2002. The Incas. Blackwell Publishers, Oxford, UK.

D'ALTROY, T.N.; LORANDI, A.M.; WILLIAMS, V.; CALDERARI, M.; HASTORF, C.; DEMARRAIS, E.; HAGSTRUM, M. 2000. Inka rule in the Northern Calchaquí valley. Argentina Journal of Field Archaeology (27): 1-26.

DÍAZ, P. P. 1981. Diario de excavación realizada en el sitio La Paya SsalCac 1. Informe depositado en el Museo Arqueológico de Cachi, Salta. MS

DILLEY, R. 1999. Ways of knowing, forms of power. Cultural Dynamics $11(1): 33-55$.

EARLE, T. y T. D'ALTROY. 1989. The political economy of the Inka Empire: the archaeology of power and finance. En: C. LambergKarlovsky (ed.), Archaeological thought in America, pp. 183-204. Cambridge University Press, Cambridge.

FERRARI, A. 2012. Espacialidad local e Inka: aportes a partir de un caso de estudio en el valle Calchaquí Norte (Salta, Argentina). Tesis para optar por el grado de Licenciado en Ciencias Antropológicas (orientación arqueológica). Facultad de Filosofía y Letras, Universidad de Buenos Aires, Argentina.

GAMARRA, LUCILA. 2008. Representando el Mundo desde un Mundo Nuevo: Trayectorias y rupturas en las prácticas funerarias y el estilo cerámico en el Valle Calchaquí Norte (Salta) durante el Período Hispano Indígena (siglo XVI-XVII). Tesis para optar por el grado de Licenciada en Antropología. Facultad de Filosofía y Letras, Universidad de Buenos Aires, MS.

GASPARINI, G. y MARGOLIES, L. 1980. Inca Architecture. Indiana University Press, Bloomington.

GIFFORD, C. 2003. Local matters: Encountering the imperial inkas in the South Andes. Ph. D. Dissertation, Columbia University, Nueva York.

GONZÁLEZ, A. R. y P. P. DÍAZ. 1992. Notas arqueológicas sobre la "Casa Morada", La Paya, pcia. de Salta. Estudios de Arqueología 5:9-61.

GONZÁLEZ, L. 2003. El oro en el Noroeste Argentino prehispánico. Estudios técnicos sobre dos objetos de la Casa Morada de La Paya. Relaciones de la Sociedad Argentina de Antropología 28: 75-99.

GOSDEN, C. 2002. Anthropology and archaeology: A changing relationship. Routledge: London and New York.

GOSDEN, C. y L. HEAD. 1994. Landscape: a usefully ambiguous concept. Archaeology in oceania 29: 113-16.

GROSBOLL, S. 1993. "...And He Said in the Time of the Ynga, They Paid Tribute and Served the Ynga". En Provincial Inca:Archaeological and ethnohistorical assessment of the impact of the Inca State, M. Malpass (Ed.), pp. 44-76. University of Iowa Press, Iowa.

HAYDEN, D. 1997. Urban landscape history: The sense of place and the politics of space. En: Understanding ordinary landscapes, Groth, P. y T. Bressi (Eds), pp. 111-133. Yale University Press, New Haven and London.

HYSLOP, J. 1984. The Inka Road System. Academic Press, New York.

HYSLOP, J. 1990. Inka settlement planning. University of Texas Press, Austin.

INGOLD, T. 2000 . The perception of the environment: essays in livelihood, dwelling and skill. Routledge, Londres.

INGOLD, T. 2010. Footprints through the weather-world: walking, breathing, knowing. Journal of the Royal Anthropological Institute 16 121-139. 
INGOLD, T. 2010b. Bringing things to life: creative entanglements in a world of materials. ERSC National Center for Research Methods, pp. 1-16.

JACOB, C. e I. LEIBOWICZ. 2011. Montañas sagradas en los confines imperiales. Nevado de Cachi, Salta-Argentina. Revista Haucayapata. Investigaciones arqueológicas del Tahuantinsuyo 2: 71-90.

KNAPPETT, C. 2005. Thinking through material culture. An interdisciplinary perspective. University of Pennsylvania Press, USA.

KOSIBA, S. 2010. Becoming Inka: The Transformation of Political Place and Practice during Inka State Formation (Cusco, Perú). Ph.D. Dissertation, Department of Anthropology, University of Chicago, Chicago.

LAVE, J. y WEGNER E. 1991. Situated learning. Legitimate peripheral participation. Cambridge University Press, Cambridge.

LEIBOWICZ, I. 2007. Espacios de poder en La Huerta, Quebrada de Humahuaca. Estudios Atacameños, Arqueología y Antropología Surandinas 34: 51-70.

LEIBOWICZ, I. 2012. Ideología y Espacio: Conquista Inka en la Quebrada de Humahuaca, Jujuy, Argentina. Revista Chilena de Antropología 25: 65-91

MACKEY, C. 2003. La transformación socioeconómica de Farfán bajo el gobierno Inka. Boletín de Arqueología PUCP 7: 321-353.

MERLEAU-PONTY, M. 1962. The Phenomenology of Perception. C Smith. Routledge and Kegan Paul, London.

MOORE, J. D. 1996. Architecture and power in the ancient Andes. The archaeology of public buildings. Cambridge University Press, Cambridge.

MORRIS, C. y J. I. SANTILLANA. 2007. The Inka transformation of the Chincha Capital. En Variations in the Expression of Inka Power, Burger, R. L., C. Morris, y R. Matos Mendieta (Eds.), pp. 135-164. Dumbarton Oaks Research Library and Collection, Washington D.C.

NIELSEN, A. E. 1995. Architectural Performance and the Reproduction of social Power. En Expanding Archaeology. Skibo, J., W. Walker y A. E. Nielsen (Eds.) 47-66. University of Utah Press, Salt Lake City.

NIELSEN, A. E. 2006. Plazas para los antepasados: Descentralización y poder corporativo en las formaciones políticas preinkaicas de los Andes circumpuneños. Estudios Atacameños (31): 63-89.
NIELSEN, A. E. y W. WALKER. 1999. Conquista Ritual y Dominación Política en el Tawantinsuyu : El Caso de Los Amarillos (Jujuy, Argentina). En Sed Non Satiata : Teoría social en arqueología Latinoamericana Contemporánea. Zarankin, A. y F. Acuto (Eds.), pp. 153-169. Ediciones del Tridente, Buenos Aires.

RAFFINO, R. A. 1978. La ocupación inka en el NO argentino: actualización y perspectivas. Relaciones de la Sociedad Argentina de Antropología VII: 95-121.

RAFFINO, R. A. 1981. Los Inkas del Kollasuyu. Ediciones Ramos Americana, La Plata, Argentina.

SERRANO, A. 1963. Lineas fundamentales de arqueología salteña. Edición del autor, Salta, Argentina.

SOJA, E. 1989. Postmodern Geographies: The Reassertion of Space in Critical Social Theory. Verso, Londres.

SPROVIERI, M. 2008 - 2009. Alucinaciones en circulación. Una mirada a la interacción surandina tardía desde las tabletas y tubos de La Paya (valle Calchaquí, Salta). Anales del Instituto de Arqueología y Etnología 63 - 64: 81 - 105. Facultad de Filosofía y Letras, Universidad Nacional de Cuyo, Mendoza.

SPROVIERI, M. y S. M. RIVERA. 2014. Las maderas de la "Colección La Paya”. Circulación y consumo en el valle Calchaquí (Salta). En Intersecciones en Antropología, (15) 1, pp. 89-102.

STRATHERN, M., 2009. Using bodies to communicate. En Social bodies. Lambert, H. y M. McDonald (Eds.), pp. 148 - 169 . Berghahn Books, USA.

TARRAGÓ, M. N. y M. DE LORENZI, 1976. Arqueología del valle Calchaquí. Etnía 23-24: 1-35.

THOMAS, J. 1993. The hermeneutics of megalithic space. En Interpretive archaeology, C. Tilley (ed.), pp. 73-98. Berg, Oxford.

THOMAS, J. 2001. Archaeologies of place and landscape. En Archaeological theory today. Hodder, I. (Ed.), pp. 165-186. Polity, Cambridge.

THOMAS, J. 2002. Archaeology's humanism and the materiality of the body. En Thinking through the body: Archaeologies of corporeality. Hamilakis Y., M. Pluciennik, y S. Tarlow (Eds), pp. 29-46. Kluwer Academic/Plenum, New York.

TURNER, S. 1994. The social theory of practices. Tradition, tacit knowledge, and presuppositions. University of Chicago Press, Chicago.

VAN DYKE, R. M. y S. ALCOCK, 2003. Archaeologies of memory: An introduction. En Archaeologies of Memory, Van Dyke, Ruth M. y Susan E. Alcock (Eds.), pp. 1-14. Blackwell, Oxford. 
VITRY, C. 2008. El Nevado de Cachi. Palloni Ediciones, Córdoba, Argentina.

WILLIAMS, V. I. 1991. Control estatal incaico en el noroeste de Argentina. Un caso de estudio: Potrero-Chaquiago. Arqueología 1: $75-103$.

WILLIAMS, V. I. 1994. Jerarquización y funcionalidad de centros estatales incaicos en el área valliserrana central. Shincal 4: 1134 .
WILLIAMS, V. I. 2000. El imperio Inka en la provincia de Catamarca. Intersecciones en antropología $\mathrm{N}^{\circ}$. Olavarría.

WILLIAMS, V. I. 2004. Poder estatal y cultura material en el Kollasuyu. Boletín de arqueología PUCP 8: 209-245.

WILLIAMS, V. I. y T. N. D'ALTROY. 1998. El sur del Tawantinsuyu. Un dominio selectivamente intensivo. Tawantinsuyu 5, Farrington, I. y R. Raffino (Eds.), pp. 170-178. Australian National University, Camberra. 